\title{
Development of a CRISPR/Cas9 genome editing toolbox for Corynebacterium glutamicum
}

\author{
Jiao Liu ${ }^{1,2+}$, Yu Wang ${ }^{1,2+}$, Yujiao Lu ${ }^{1,2}$, Ping Zheng ${ }^{1,2^{*}}$, Jibin Sun ${ }^{1,2^{*}}$ and Yanhe Ma ${ }^{2}$
}

\begin{abstract}
Background: Corynebacterium glutamicum is an important industrial workhorse and advanced genetic engineering tools are urgently demanded. Recently, the clustered regularly interspaced short palindromic repeats (CRISPR) and their CRISPR-associated proteins (Cas) have revolutionized the field of genome engineering. The CRISPR/Cas9 system that utilizes NGG as protospacer adjacent motif (PAM) and has good targeting specificity can be developed into a powerful tool for efficient and precise genome editing of C. glutamicum.
\end{abstract}

Results: Herein, we developed a versatile CRISPR/Cas9 genome editing toolbox for C. glutamicum. Cas9 and gRNA expression cassettes were reconstituted to combat Cas9 toxicity and facilitate effective termination of gRNA transcription. Co-transformation of Cas9 and gRNA expression plasmids was exploited to overcome high-frequency mutation of cas9, allowing not only highly efficient gene deletion and insertion with plasmid-borne editing templates (efficiencies up to 60.0 and 62.5\%, respectively) but also simple and time-saving operation. Furthermore, CRISPR/Cas9-mediated ssDNA recombineering was developed to precisely introduce small modifications and single-nucleotide changes into the genome of C. glutamicum with efficiencies over $80.0 \%$. Notably, double-locus editing was also achieved in C. glutamicum. This toolbox works well in several C. glutamicum strains including the widely-used strains ATCC 13032 and ATCC 13869.

Conclusions: In this study, we developed a CRISPR/Cas9 toolbox that could facilitate markerless gene deletion, gene insertion, precise base editing, and double-locus editing in C. glutamicum. The CRISPR/Cas9 toolbox holds promise for accelerating the engineering of C. g/utamicum and advancing its application in the production of biochemicals and biofuels.

Keywords: CRISPR/Cas9, Genome editing, Corynebacterium glutamicum, Plasmid-borne templates, Gene deletion/ insertion, Single-nucleotide editing, Double-locus editing

\section{Background}

The Gram-positive soil bacterium Corynebacterium glutamicum was discovered about 60 years ago, and was originally well-known as an excellent producer of glutamate [1]. With the development of biotechnology, $C$. glutamicum has been successfully engineered to serve as a versatile workhorse for industrial bioproduction. Nowadays, this bacterium is used to produce more than

\footnotetext{
*Correspondence: zheng_p@tib.cas.cn; sun_jb@tib.cas.cn

†Jiao Liu and Yu Wang contributed equally to this work

${ }^{2}$ Tianjin Institute of Industrial Biotechnology, Chinese Academy of Sciences, Tianjin 300308, People's Republic of China

Full list of author information is available at the end of the article
}

4 million tons of diverse amino acids per year and a wide range of other natural and non-natural products, which are used as feed additives, nutritional supplements, pharmaceutical intermediates, biofuels, and polymer building blocks [2]. It is estimated that products generated via C. glutamicum fermentation will reach a market size of US $\$ 20.4$ billion by 2020 [3].

At the early stage of engineering of C. glutamicum, random mutagenesis combined with positive selection by phenotypic resistance to amino acid analogs was the most commonly used strategy [4]. Genetic manipulations in C. glutamicum were initiated in 1984 and became a key enabling strategy for strain improvement [5]. A routinely used method for gene disruption and insertion in 
C. glutamicum is based on integration of a suicide vector into its chromosome, followed by a second recombination event to remove the plasmid backbone and a counter-selection step using a conditionally lethal marker. Nevertheless, due to the frequent spontaneous inactivation of the counter-selectable marker $s a c B$, up to $45 \%$ of colonies obtained in the screening process were falsepositive, making this multi-step procedure time-consuming and inefficient [6].

Recently, clustered regularly interspaced short palindromic repeats (CRISPR) and their CRISPR-associated proteins (Cas) have been explored as a leading-edge tool for genome editing in eukaryotic host cells, plants, and animal models of human disease [7-9]. Although CRISPR/Cas systems (especially CRISPR/Cas9 systems) are derived from bacteria or archaea, their applications have not been extensively employed in bacteria [10]. To date, successes of bacterial genome editing using CRISPR/Cas9 systems have been reported in limited bacteria including Escherichia coli [11-19], Streptococcus species [11, 20], Lactobacillus reuteri [21], Streptomyces species [22-24], Tatumella citrea [12], Clostridium species [25-29], Bacillus subtilis [30-32], Myceliophthora species [33], and Synechococcus elongates [34].

To engineer C. glutamicum more efficiently and unleash its potential in industrial biotechnology, facile yet robust genome editing tools are urgently demanded. The CRISPR/Cas9 system that utilized NGG as protospacer adjacent motif (PAM) and has good targeting specificity is expected to enable genome-wide scale and precise editing of GC-rich C. glutamicum [10]. To our knowledge, there were many tries in the community to adapt CRISPR/Cas9 into C. glutamicum for comprehensive genome editing. In the time this manuscript was being prepared, Cho and coworkers reported CRISPR/ Cas9-mediated genome editing of C. glutamicum using cas 9 gene codon-optimized for its use in actinomycetes and $\operatorname{rec} T$ gene encoding $E$. coli prophage recombinase [35]. Without the use of $\operatorname{rec} T$, no positive transformants could be obtained using double-stranded DNA (in both linear and replicative plasmid form) or single-strand DNA (ssDNA) as editing templates. Further introduction of $\operatorname{rec} T$ facilitated deletion of 400-bp chromosomal fragments using ssDNA as editing templates. However, Cho and coworkers didn't report whether the optimized CRISPR/Cas9 system including RecT could delete or insert genes into the chromosome of $C$. glutamicum with plasmid-borne templates [35]. Meanwhile, Jiang and coworkers independently developed a CRISPR/Cpf1mediated genome editing tool for C. glutamicum [36]. The CRISPR-Cpf1 system combined with ssDNA recombineering can efficiently introduce small changes into the C. glutamicum genome. Large gene deletions and insertions are also realizable using this system [36]. However, because Francisella novicida Cpf1 utilizes a T-rich PAM [37], its editing targets in GC-rich C. glutamicum genome are supposed to be fewer than Streptococcus pyogenes Cas9. Therefore, development of a powerful CRISPR/Cas9-mediated genome editing toolbox that can delete, insert and modify genes in C. glutamicum flexibly and multiply is still urgently demanded.

In this study, we successfully developed a CRISPR/ Cas9 toolbox for efficient and comprehensive engineering of several C. glutamicum strains. By using the tailormade CRISPR/Cas9 system, gene deletion and insertion with plasmid-borne editing templates were achieved with efficiencies of $30.8-60.0 \%$ and $16.7-62.5 \%$, respectively. CRISPR/Cas9-mediated ssDNA recombineering was developed to introduce small modifications and single-nucleotide changes into the genome with efficiencies over $80.0 \%$. Double-locus editing was also realized in C. glutamicum with an efficiency of $40.0 \%$. The toolbox developed here is simple and versatile, which will provide solutions to overcome major limitations of existing genome editing technologies of C. glutamicum and advance engineering and application of this industrial workhorse.

\section{Results \\ Optimization of Cas9 and gRNA expression for lethality-based selection}

It has been repeatedly reported that the double-strand breakage (DSB) induced by Cas9 is lethal to bacterial cells because many microorganisms lack the endogenous nonhomologous end joining (NHEJ) mechanism, or the NHEJ is not efficient enough to repair the DSB. As a result, CRISPR/Cas9 was usually used as a lethalitybased selection tool in bacterial cells $[25,26]$. To achieve CRISPR/Cas9-mediated genome editing, the lethality of Cas9-induced DSB was first evaluated.

Constitutive expression of dCas9 from S. pyogenes in C. glutamicum has been proven unattainable [3]. In a previous study, isopropyl- $\beta$-D-thiogalactopyranoside (IPTG)-inducible promoter $P_{t a c}$ and propionate-inducible promoter $P_{p r p D 2}$ were used for dCas9 expression, and $P_{t a c}$ was used for gRNA expression, facilitating CRISPR interference (CRISPRi) in C. glutamicum [3]. Inspired by this study, we used the same biological parts to prepare Cas9 and gRNA expression cassettes. Strictly controlled expression of Cas9 was considered beneficial for screening desirable mutants when using CRISPR/ Cas9 for gene deletion [26]. To optimize the promoter for Cas9 expression, $P_{t a c}$ and $P_{p r p D 2}$ were employed to drive the expression of a Cas9-red fluorescent protein (RFP) fusion protein that consisted of the first $180 \mathrm{bp}$ of cas 9 gene and the full-length $r f p$ gene. The artificial 
$P_{t a c} / P_{p r p D 2}-c a s 9^{180 \mathrm{bp}}-r f p$ cassettes were inserted into pXMJ19, generating plasmids pRfp1 and pRfp2, respectively (Fig. 1a). Fluorescence outputs of the engineering cells harboring pRfp1 and pRfp2 were detected in the presence or absence of inducers. The results demonstrated that $P_{t a c}$ was controlled more strictly by IPTG

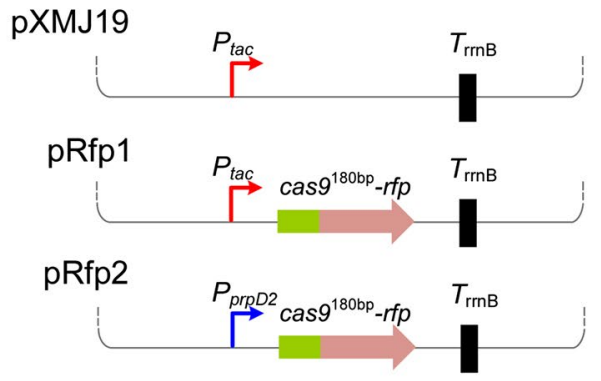

C

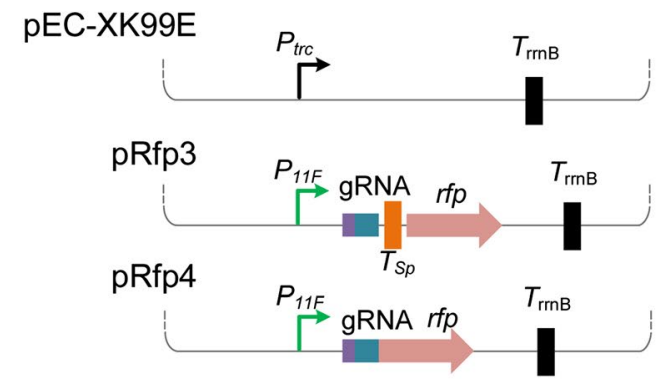

e

Cas9 handle (42 nt)

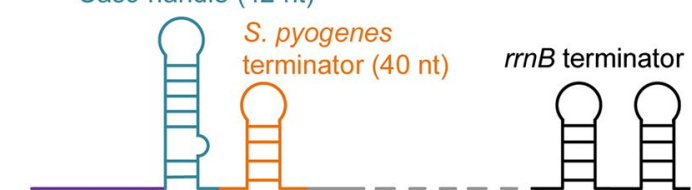

Base-pairing region (20-25 nt)
Redundant segment (185 nt) b

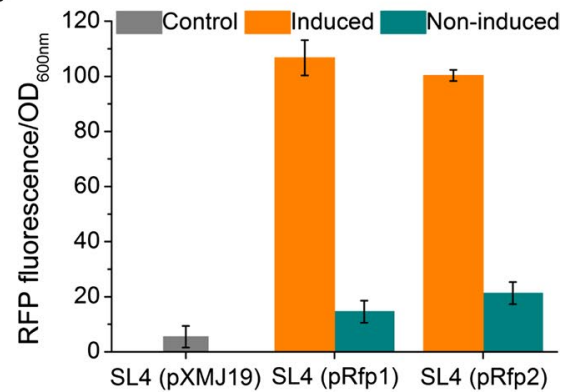

d

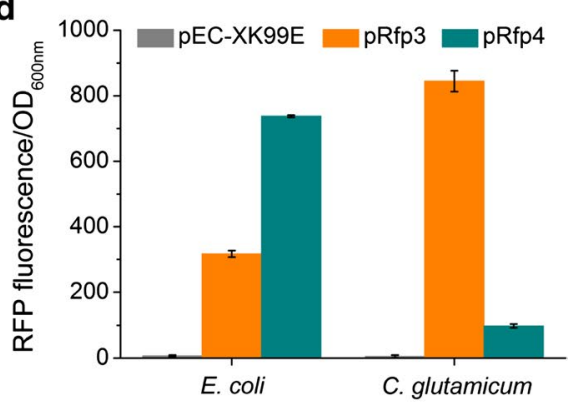

f

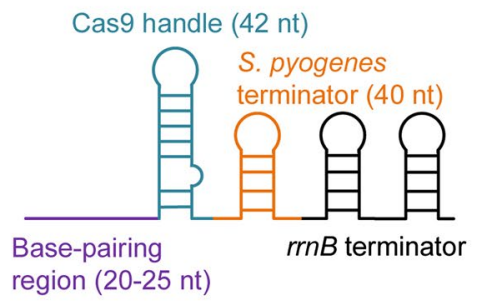

g

\begin{tabular}{ll}
\hline Strain & Escape rate $^{\mathrm{a}}$ \\
\hline SL4 (pCas9+pgRNA1) & $8.7 \pm 1.9 \times 10^{-2}$ \\
SL4 (pCas9+pgRNA2) & $1.3 \pm 0.2 \times 10^{-2}$ \\
\hline
\end{tabular}

${ }^{a}$ Average of three independent experiments.

Fig. 1 Optimization of CRISPR/Cas9 system in C. glutamicum. a Schematic representation of the plasmids used for optimizing the promoter of Cas9 expression. The cas $9^{180 \mathrm{bp}}$-rfp fusion gene that consisted of the first $180 \mathrm{bp}$ of cas 9 gene and the full-length $r f p$ gene was inserted into pXM 19 under the control of IPTG-inducible promoter $P_{\text {tac }}$ (pRfp1) or propionate-inducible promoter $P_{\text {prpD2 }}$ (pRfp2). The empty plasmid pXMJ19 was used as a negative control. b Optimization of promoters for Cas9 expression. pRfp1, pRfp2, and pXMJ19 were transformed into C. glutamicum SL4 separately. The resultant transformants were cultivated in SGY medium with or without $1 \mathrm{mM} \mathrm{IPTG} \mathrm{(for} \mathrm{pRfp1)} \mathrm{or} 1 \mathrm{~g} / \mathrm{L}$ sodium propionate (for pRfp2). Cells of the stationary growth phase were used to detect their fluorescence outputs using a microplate reader $(\lambda$ excitation $=560 \mathrm{~nm}, \lambda$ emission $=607 \mathrm{~nm}$ ). c Schematic representation of the plasmids used for verifying the function of the terminator derived from $S$. pyogenes $\left(T_{S p}\right)$. A rfp gene was inserted downstream the gRNA (pRfp3) and the modified gRNA with $T_{\text {sp }}$ deleted (pRfp4). The empty plasmid pEC-XK99E was used as a negative control. d Function verification of $T_{s p}$ in E. coli and C. glutamicum by detecting fluorescence outputs of strains harboring pRfp3, pRfp4, or pEC-XK99E. e The gRNA structure derived from plasmid pgRNA1. f The optimized gRNA structure derived from plasmid pgRNA2. g Escape rate of CRISPR/Cas9-based counter-selection using different gRNA expression plasmid. pgRNA1 and pgRNA2 were transformed into C. glutamicum SL4 (pCas9) separately. Correct transformants were cultivated, diluted and spread on SGY plates containing Km and Cm, with or without IPTG (1 mM). The escape rate of counter-selection was calculated by colony counting 
than $P_{p r p D 2}$ by sodium propionate (Fig. 1b). Plasmid pCas9 was then constructed by inserting $P_{t a c}$-cas 9 cassette into pXMJ19 and transformed into C. glutamicum SL4, a derivative of strain ATCC 13869 with high electrotransformation efficiency, generating strain SL4 (pCas9). The gRNA targeting on the C. glutamicum lactate dehydrogenase gene $l d h A$ was designed and inserted into pEC-XK99E under the control of $P_{t a c}$, resulting in pgRNA1. Transformation of pgRNA1 into strain SL4 (pCas9) was performed and cells were spread on soya peptone-glucose-yeast extract (SGY) plates containing kanamycin $(\mathrm{Km})$ and chloramphenicol $(\mathrm{Cm})$ with or without $1 \mathrm{mM}$ IPTG. All the colonies growing on the plates were counted to calculate the escape rate. A high escape rate $\left(8.7 \pm 1.9 \times 10^{-2}\right)$ was obtained, which was unacceptable for lethality-based selection.

The unoptimized gRNA expression cassette may be one reason for the high escape rate of CRISPR/Cas9-based counter-selection. Considering that cas 9 and gRNA were both under control of $P_{t a c}, P_{11 F}$ which is a derivative of the strong constitutive promoter $P_{c s p B}$ of $C$. glutamicum [38] was recruited for gRNA expression to avoid possible interference between the two $P_{t a c}$ promoters. The secondary structure of gRNA is also crucial for forming Cas9gRNA complex. The gRNA used here consists of three domains: a 20-25 nt complementary region for specific DNA binding, a 42 nt hairpin for Cas9 binding (Cas9 handle), and a $40 \mathrm{nt}$ transcription terminator derived from $S$. pyogenes $\left(T_{S p}\right)$ [39]. $T_{S p}$ functioned well in $E$. coli and many other bacteria $[26,30,39]$. It was also recruited to develop CRISPRi in C. glutamicum, but its termination ability has not been investigated in C. glutamicum [3]. To evaluate $T_{S p}$, a $r f p$ gene was inserted into the downstream of gRNA, producing plasmid pRfp3. $T_{S p}$ of pRfp3 was further deleted, resulting in plasmid pRfp4 (Fig. 1c). As expected, deletion of $T_{S p}$ increased $r f p$ expression in $E$. coli, demonstrating that $T_{S p}$ was functional in $E$. coli. However, the $r f p$ downstream $T_{S p}$ was highly expressed in C. glutamicum and deletion of $T_{S p}$ decreased $r f p$ expression (Fig. 1d). This surprising phenomenon suggests that $T_{S p}$ does not function as a terminator in $C$. glutamicum. In plasmid pgRNA1, $T_{S p}$ was followed by a $185 \mathrm{bp}$ redundant segment and a $r r n B$ terminator $\left(T_{r r n B}\right)$. Since $T_{S p}$ could not terminate gRNA transcription in $C$. glutamicum, the $185 \mathrm{bp}$ redundant segment will also be transcribed, which may affect the function of Cas9-gRNA complex (Fig. 1e). To confirm our hypothesis, the $185 \mathrm{bp}$ redundant segment was removed from plasmid pgRNA1. The resultant plasmid pgRNA2 harbored a gRNA expression cassette in which $T_{r r n B}$ closely followed $T_{S p}$ (Fig. 1f). Lethality test using pCas9 and pgRNA2 showed that the escape rate was reduced to $1.3 \pm 0.2 \times 10^{-2}$, making the CRISPR/Cas9-based counter-selection feasible (Fig. 1g).
Markerless chromosomal gene deletion using CRISPR/Cas9 and plasmid-borne editing template

Since the CRISPR/Cas9 system could act as an efficient selection tool, we exploited its capacity for markerless chromosomal gene deletion. Two $\sim 1000$ bp homologous arms flanking at both sides of the targeted $l d h A$ gene were inserted into plasmid pgRNA2. The resultant plasmid pgRNA3 was then transformed into C. glutamicum SL4 (pCas9) to provide a DNA editing template for deleting $664 \mathrm{bp}$ of $l d h A$ gene. It was expected that edited mutants would be generated through double-crossover homologous recombination and nonedited cells would be eliminated by CRISPR/Cas9-directed cleavage at the targeted ldhA gene (Fig. 2a). Noteworthily, the transformants obtained could be divided into two categories, abnormally large ones and small ones (Fig. 2b). Colony PCR was conducted to confirm gene deletion in the transformants using a pair of primers shown in Fig. 2a. All the abnormally large colonies were false and two of the twenty small ones tested were positive mutant (Additional file 1: Figure S1).

Although successful gene deletion was achieved by using CRISPR/Cas9 system, the editing efficiency was quite low. We speculated that two events might help the false positives escape the counter-selection. First, the targeted DNA was cleaved by Cas9 but the DSB was repaired by nucleotide or base excision repair, leading to the survival of cells and very likely some mutations at the cleavage site [40]. In this case, the targeted gene might be deactivated but PCR was not capable of verifying it. Second, pCas9 or pgRNA3 mutated, which disabled the Cas9-gRNA complex and the counter-selection system. To confirm our hypotheses, the $l d h A$ genes of false positives were amplified by PCR and sequenced. pCas 9 and pgRNA3 were also extracted from the false positives and sequenced. No mutation was found in gene $l d h A$ or plasmid pgRNA3, whereas plasmid pCas9 mutated in several patterns as shown in Fig. 2c. In all the abnormally large colonies investigated, their cas 9 genes were all deleted from pCas9 plasmids. In the small but false colonies, their cas9 genes were either deactivated through nonsense mutation or disrupted by insertion of a transposase encoding gene from $E$. coli. Such cells harboring mutated pCas9 escaped the counter-selection, and even worse, outgrew those expressing active Cas9, making selection of the edited cells very difficult.

Since cas 9 was easily mutated in C. glutamicum, reducing replication of pCas9 in the host may lessen the mutation. A one-step electrotransformation strategy was then presented that pCas9 and pgRNA3 were co-transformed into C. glutamicum SL4 simultaneously and cells were spread on SGY plates supplemented with $\mathrm{Km}, \mathrm{Cm}$, and IPTG (1 mM) immediately after recovery. Screening 
a

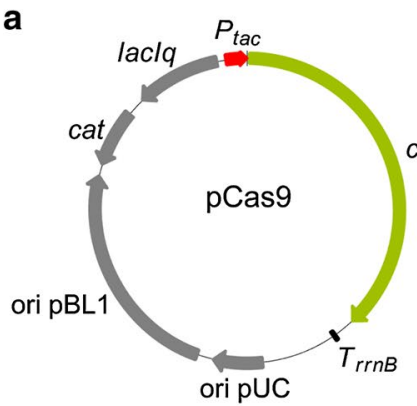

$\operatorname{cas} 9$

oripuc

Wild-type

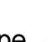
downstréam

Mutant

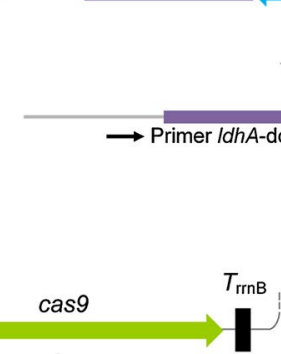
Idh

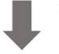

C

pCas 9

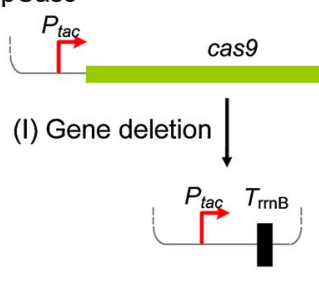

d

\begin{tabular}{lllll}
\hline Replicate & Total colony & Big colony & Small colony & Editing efficiency \\
\hline 1 & 10 & 2 & 8 & $60.0 \%(6 / 10)$ \\
2 & 11 & 5 & 6 & $36.4 \%(4 / 11)$ \\
3 & 11 & 2 & 9 & $45.5 \%(5 / 11)$ \\
\multicolumn{2}{l}{ Average } & & & $47.3 \pm 11.9 \%$ \\
\hline
\end{tabular}

(II) Nonsense mutation

(III) Insertional inactivation

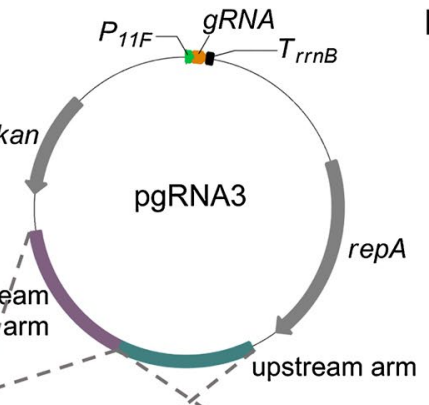

b

Cas9-gRNA

$\longleftarrow$ Primer IdhA-up
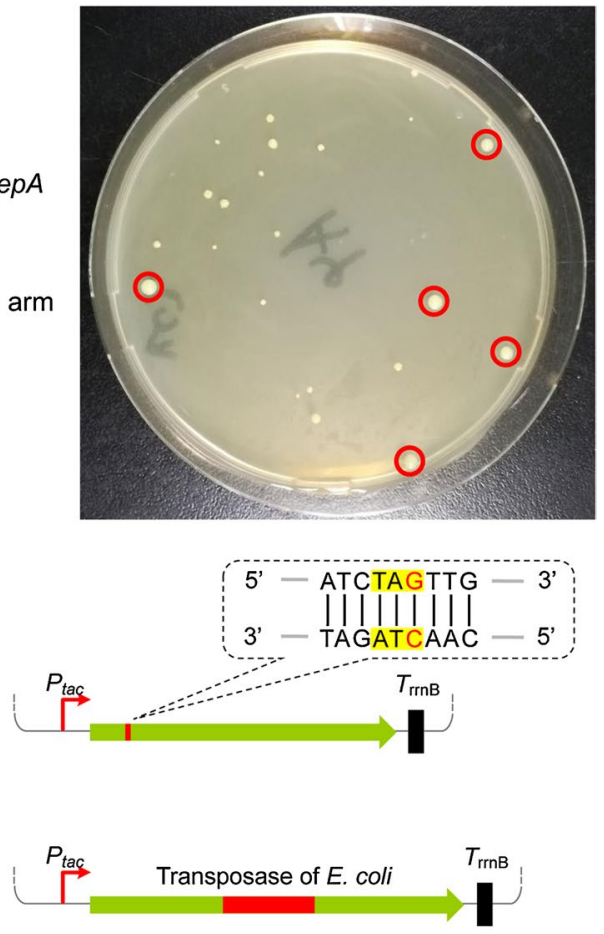

e

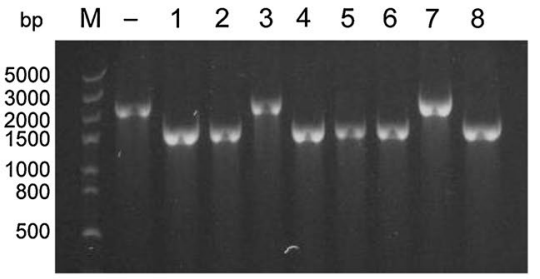

Fig. 2 Gene deletion using CRISPR/Cas9 and plasmid-borne editing template in C. glutamicum SL4. a Schematic representation of pCas9, pgRNA3, and gene deletion event. b Transformants harboring pCas9 and pgRNA3 on a SGY plate supplemented with Km, Cm, and IPTG (1 mM). pCas9 and pgRNA3 were co-transformed into strain SL4 simultaneously and cells were spread on SGY plates with Km, Cm, and IPTG (1 mM) immediately after recovery. Colonies marked in red cycles were the so-called abnormally large colonies. c Schematic representation of different patterns of pCas 9 mutation. Gene deletion, cas9 gene was removed from pCas9. Nonsense mutation, a T465G mutation occurred, generating a stop codon (TAT to TAG) in cas 9 gene. Insertional inactivation, a transposase encoding gene from E. coli was inserted into cas 9 gene, which deactivated cas9. d Colony counting and editing efficiency calculation of C. glutamicum SL4. Colonies on SGY plates supplemented with Km, Cm, and IPTG (1 mM) were counted and verified using colony PCR. e PCR verification of IdhA deletion in C. glutamicum SL4 using the primer pair (IdhA-up and IdhA-down) shown in a. M, DNA marker; -, wild-type control; 1-8, eight small colonies. This displayed the result of Replicate 1 in c. The results of Replicate 2 and Replicate 3 were shown in Additional file 1: Figure $\mathbf{S 2}$

using two antibiotics and expressing cas 9 may burden cell growth, and colonies appeared after 2-3 days cultivation. We still got some abnormally large colonies and they were all proven false by colony PCR. However, the ratio of positive mutants out of all colonies obtained in one plate considerably increased, reaching an average of $47.3 \pm 11.9 \%$ (Fig. 2d, e; Additional file 1: Figure S2). Given the high editing efficiency, edited cells should be easily obtained after CRISPR/Cas9 counter-selection.
Meanwhile, a control experiment was conducted using pCas9 and pgRNA3-derivative plasmid that harbored no targeting spacer. As expected, no counter-selection phenomenon was observed and no gene deletion mutants were obtained (Additional file 1: Figure S3).

\section{Markerless chromosomal gene insertion}

Next, the ability of gene insertion (allelic exchange) by using CRISPR/Cas9 system was examined. A $r f p$ cassette 
(781 bp) was inserted between the upstream and downstream homologous arms of pgRNA3. The resultant plasmid pgRNA4, together with pCas9, was co-transformed into C. glutamicum SL4, aiming to replace the ldhA gene with the $r f p$ cassette ( $\Delta 664 \mathrm{bp}$ and insert $781 \mathrm{bp}$ ) (Fig. 3a). Colony PCR was conducted to verify the gene insertion events by using a pair of primers shown in Fig. 3a. An average of $25.0 \pm 8.3 \%$ of colonies were confirmed as positive mutants (Fig. 3b, c; Additional file 1: Figure S4). The mutant inserted with the $r f p$ cassette in the $l d h A$ gene was designated as C. glutamicum SL4AldhA::rfp.

\section{ssDNA-directed recombineering using CRISPR/Cas9 and RecT}

ssDNA-directed recombineering can be used to mutagenize, repair or engineer the chromosome with high efficiencies [41]. By introducing exogenous recombinases into $C$. glutamicum, the recombination efficiency using ssDNA templates was much higher than that using plasmid-borne templates [42]. However, due to lack of reliable genotype selection method, application of this technique was severely limited. Taking advantage of the
CRISPR/Cas9 system developed here, counterselecting mutants generated during recombineering against wild-type cells became feasible and a ssDNA-directed gene editing tool was developed. The $\operatorname{rec} T$ gene encoding recombinase RecT of prophage Rac was inserted into plasmid pgRNA4 under the control of the propionateinducible promoter $P_{p r p D 2}$, resulting in plasmid pgRNA5 (Fig. 4a). ssDNAs were designed to alter the PAM sequence or PAM-proximal 8-12 bp sequences because these regions were shown to be most crucial for Cas9 targeting specificity [43]. To obtain high recombination efficiency, 90mer ssDNAs targeted to the lagging strand were used here.

The method was first tested for the ability to introduce stop codons into a target gene. A ssDNA (rfp-off1, Additional file 1: Table S1) was designed to introduce two successive stop codons into the $r f p$ gene of $C$. glutamicum SL4 $\Delta l d h A:: r f p$ (Fig. 4b). The operation scheme was shown in Fig. 4b. Plasmid pgRNA5 was first transformed into strain SL4 $\Delta l d h A:: r f p$. The resultant strain SL4 $\Delta l d h A:: r f p$ (pgRNA5) was cultivated with addition of sodium propionate to induce RecT expression, and then
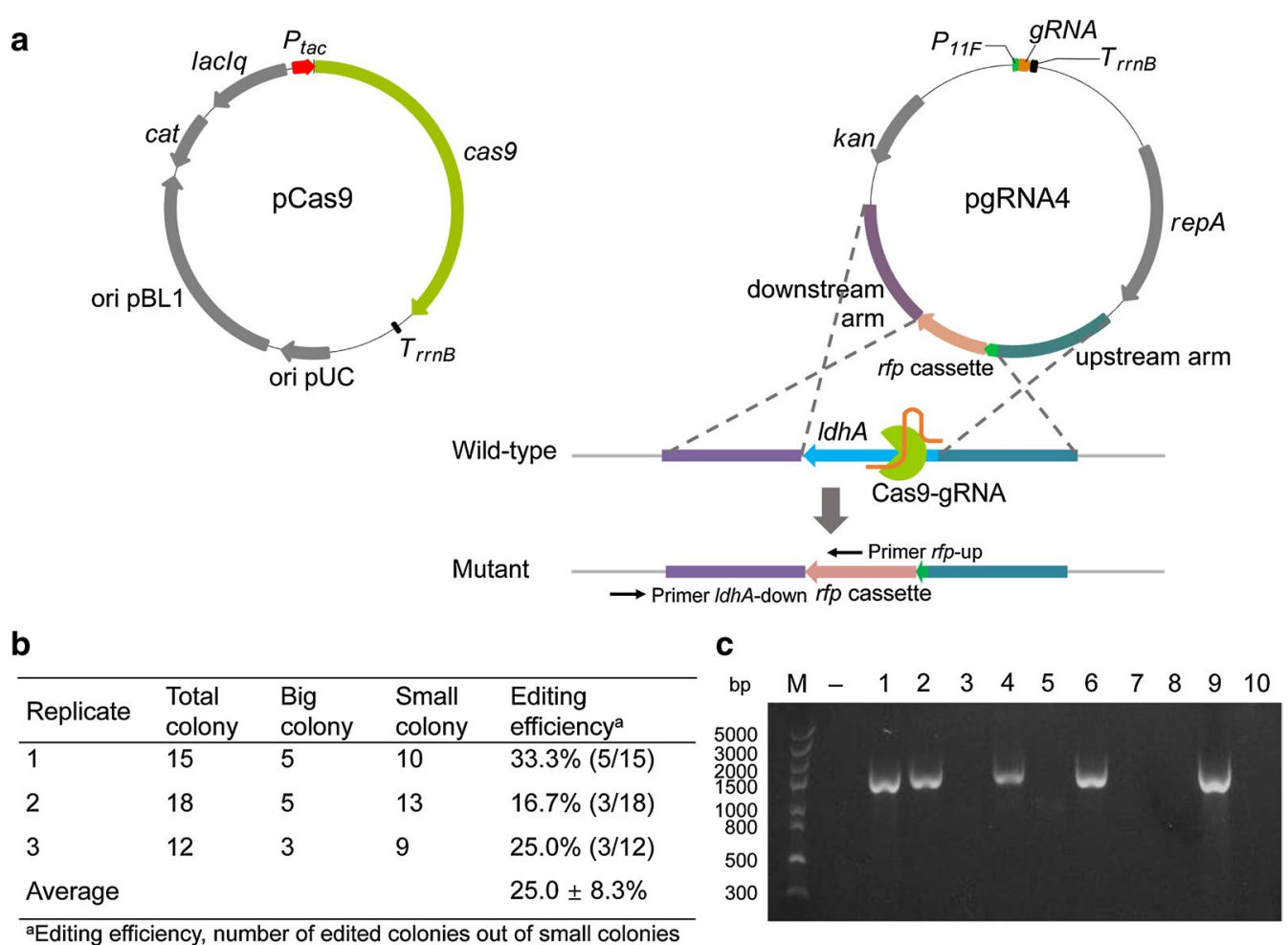

Fig. 3 Gene insertion using CRISPR/Cas9 and plasmid-borne editing template in C. glutamicum SL4. a Schematic representation of pCas9, pgRNA4, and gene insertion event. b Colony counting and editing efficiency calculation of C. glutamicum SL4. pCas9 and pgRNA4 were co-transformed into C. glutamicum SL4 simultaneously and cells were spread on SGY plates supplemented with Km, Cm, and IPTG (1 mM) immediately after recovery. Colonies on the selective plates were counted and verified using colony PCR. C PCR verification of rfp insertion in C. glutamicum SL4 using the primer pair (rfp-up and IdhA-down) shown in a. M, DNA marker; -, wild-type control; 1-10, ten small colonies. This displayed the result of Replicate 1 in $\mathbf{b}$. The results of Replicate 2 and Replicate 3 were shown in Additional file 1: Figure S4 


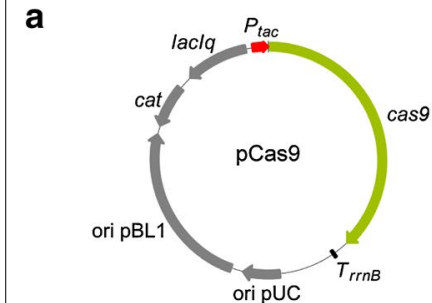

C

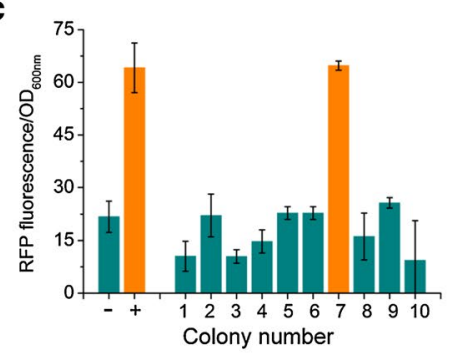

e

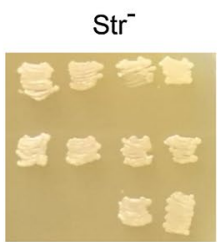

$\operatorname{Str}^{+}$

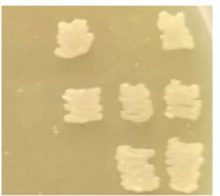

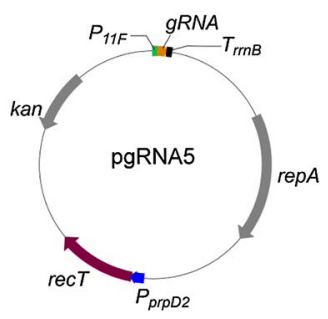

d

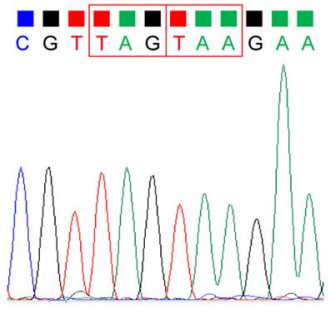

f C C C TCG CA A G C C

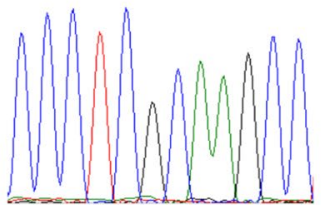

b
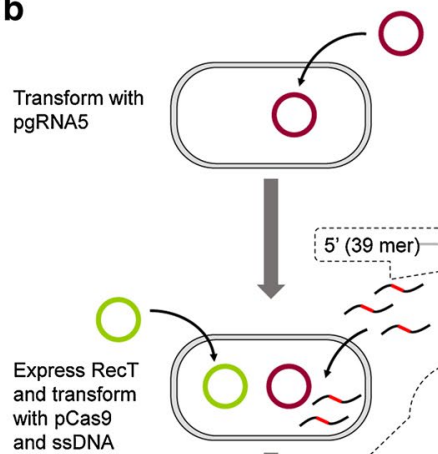

and SSDNA

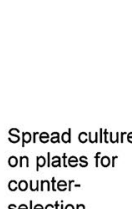

selection

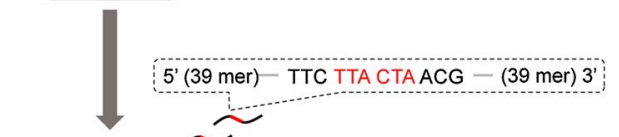

Detecting
RFP output

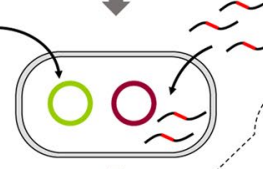

Strain SL4 $\triangle$ /dhA::Ifp

5' - CGT CCG TAC GAA - 3' II $111 \| 11111$

3' - GCA GGC ATG CTT - 5'

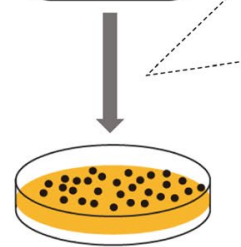

Strain SL4 $/$ /dhA::rfpoff 5' - CGT TAG TAA GAA - 3 ' || ||| || || 3' - GCAATC ATT CTT - 5'

Fig. 4 ssDNA-directed recombineering using CRISPR/Cas9 and RecT in C. glutamicum SL4 $\triangle$ IdhA::rfp. a Schematic representation of pCas9 and pgRNA5 which were used for ssDNA-directed recombineering. $\mathbf{b}$ Operation scheme of ssDNA-directed recombineering using CRISPR/Cas9 and RecT for rfp deactivation. A 90mer ssDNA targeted to the lagging strand (rfp-off1, Additional file 1: Table S1) is designed to introduce two successive stop codons (highlighted in red) in rfp gene. PAM sequence of the gRNA is shaded grey. Plasmid pgRNA5 was first transformed into C. glutamicum

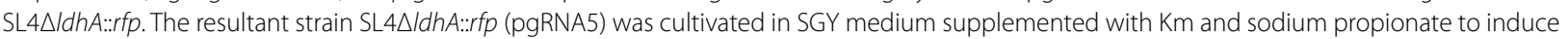
RecT expression. Electrocompetent cells were then prepared and transformed with pCas 9 and ssDNA. After recovery, cells were spread on SGY plates supplemented with $\mathrm{Km}, \mathrm{Cm}$, and IPTG $(1 \mathrm{mM})$ to induce Cas9 expression for counter-selection. Colonies were picked randomly and verified

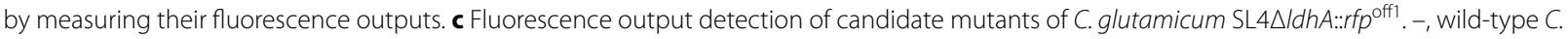
glutamicum SL4 control; +, C. glutamicum SL4 $\triangle / d h A: \cdot r f p ; 1-10$, ten colonies. This displayed the result of Replicate 1. The results of Replicate 2 and Replicate 3 were shown in Additional file 1: Figure S6. d rfp gene sequencing of C. glutamicum SL4 $\triangle /$ dhA::rfpoff1 mutants. Nucleotides in red box represents the two successive stop codons (TAG TAA) introduced by ssDNA-directed recombineering. e Str ${ }^{R}$ phenotype test of C. glutamicum SL4 $r p s{ }^{\mathrm{K} 43 \mathrm{R}}$ mutants. Ten colonies were picked randomly from SGY plates supplemented with $\mathrm{Km}, \mathrm{Cm}$, and IPTG (1 mM) and patched onto SGY plates $\left(\mathrm{Str}^{-}\right)$and SGY plates supplemented with streptomycin $\left(\mathrm{Str}^{+}\right)$. Seven out of ten colonies were $\mathrm{Str}{ }^{\mathrm{R}}$. $\mathbf{f} r p s L$ gene sequencing of $C$. glutamicum SL4 $r p s L^{K 43 R}$ mutants. Nucleotides in red box represents the mutated codon (AAG to CGC) introduced by ssDNA-directed recombineering

electrocompetent cells were prepared. Next, cells were co-transformed with pCas9 and ssDNA, recovered, and spread on SGY plates supplemented with $\mathrm{Km}, \mathrm{Cm}$, and IPTG $(1 \mathrm{mM})$ to induce Cas9 expression. Three independent experiments were conducted and hundreds of colonies could be obtained in each experiment, which were much more than the number of colonies got in gene deletion and insertion experiments (Additional file 1: Figure S5). For each replicate, 10 colonies were picked randomly and verified by measuring their fluorescence outputs, (Fig. 4c; Additional file 1: Figure S6). The gene editing events were further verified by gene sequencing (Fig. 4d), demonstrating a high editing efficiency of $86.7 \pm 5.8 \%$, an average of $90.0,90.0$, and $80.0 \%$ from three replicates. Such high mutation rate would eliminate the need of phenotype screening methods for ssDNAdirected recombineering, making genome editing of $C$. glutamicum more efficient.

Ribosomal protein S12 encoding gene rpsL was selected as another target for ssDNA-directed gene editing using CRISPR/Cas9. An amino acid mutation K43R (AAG to CGC in the nucleotide sequence) resulting in resistance to streptomycin $\left(\mathrm{Str}^{\mathrm{R}}\right)$ was described for C. glutamicum $[44,45]$. A ssDNA was designed to mutate AAG to CGC which corresponded to amino acid mutation K43R, and plasmid pgRNA6 was then constructed. Using the protocol described previously, mutants with $\operatorname{Str}^{R}$ phenotype were obtained with a mutant efficiency of $70.0 \%$ (Fig. 4e). 
Gene sequencing further verified the targeted gene editing of $r p s L$ (Fig. 4f).

\section{Plasmid curing}

To cure the Cas9 and gRNA expression plasmids for continuous genome editing, engineered cells were cultivated in SGY medium without antibiotics for $12 \mathrm{~h}$. The culture was serially transferred into fresh medium for another two times before plating on SGY plates without antibiotics. The resultant colonies were picked and tested for resistance to $\mathrm{Km}$ and $\mathrm{Cm}$. Approximately $25.0 \%$ of colonies were sensitive to $\mathrm{Km}$ and $\mathrm{Cm}$, confirming that both plasmids were cured. In the study that reported CRISPR/ Cpf1-mediated genome editing of C. glutamicum, Jiang et al. replaced the replicon of pXM)19 (cas9 expression plasmid) with a temperature sensitive replicon $\mathrm{pBL}^{\text {ts }}$ [46] and deleted the distribution protein Per encoding gene [47] in pEC-XK99E (gRNA expression plasmid), facilitating easy plasmid curing [36]. Since plasmids with the same backbones were used in our and Jiang et al's studies [36], the strategy reported by Jiang et al. is also applicable to optimizing the present plasmid curing procedure.

\section{CRISPR/Cas9-mediated genome editing in C. glutamicum wild-type strains}

To evaluate the universality of the CRISPR/Cas9mediated genome editing toolbox described above, its application was tested in two well-known wild-type $C$. glutamicum strains ATCC 13869 and ATCC 13032. ldhA gene knockout was first performed. After co-transformation of pCas9 and pgRNA3, cells were spread on SGY plates supplemented with $\mathrm{Km}, \mathrm{Cm}$, and $1 \mathrm{mM}$ IPTG. However, the cell growth was severely hindered. Colonies of strain ATCC 13869 appeared after 8 days cultivation and no colonies of strain ATCC 13032 were obtained even after 10 days cultivation. Considering that expression of Cas9 can be a significant burden for cell growth, we hypothesized that the expression level of Cas9 was too high for strains ATCC 13969 and ATCC 13032. Therefore, the inducer (IPTG) usage was reduced and $0.1 \mathrm{mM}$ IPTG and $0.01 \mathrm{mM}$ IPTG were proven suitable for strains ATCC 13969 and ATCC 13032, respectively. After 2-3 days cultivation, colonies were picked for verification, revealing that $l d h A$ deletion efficiencies reached $33.3 \pm 2.5$ and $60.0 \%$ in strains ATCC 13969 and ATCC 13032, respectively (Table 1). When shorter homologous arms ( $500 \mathrm{bp})$ were used to knock out ldhA in strain ATCC 13032, fewer colonies but comparable deletion efficiency $(50.0 \%)$ were obtained (Table 1 ), indicating that shorter homology arms may result in lower homologous recombination efficiencies. An 8083 bp fragment (cgl1776-cgl1781) was selected as a second target for CRISPR/Cas9-mediated gene deletion in strain ATCC 13032. Using plasmids pCas9 and pgRNA9 harboring $1000 \mathrm{bp}$ homologous arms, the $8083 \mathrm{bp}$ fragment was successfully deleted with an efficiency of $40.0 \%$ (Table 1).

By using plasmids pCas9 and pgRNA4, replacement of $l d h A$ gene with the $r f p$ cassette was conducted in strains ATCC 13969 and ATCC 13032. The editing efficiencies reached 28.6 and $62.5 \%$, respectively (Table 1 ). Then, in order to assess the possibility of inserting larger DNA fragments into the genome of C. glutamicum using CRISPR/Cas9, we attempted to insert a 3626 bp lacZ cassette into the genomic locus between cgl0900 and cgl0901 without deleting any chromosomal fragment. By co-transforming pCas9 and pgRNA10 into strain ATCC 13032 , insertion of the lac $Z$ cassette was achieved at an efficiency of $50.0 \%$ (Table 1). These results demonstrate the possibility of deleting and inserting larger DNA fragments in C. glutamicum.

By using pCas9, pgRNA5 and ssDNA $r f p$-off1 (Additional file 1: Table S1), ssDNA-directed recombineering was also successfully performed in C. glutamicum ATCC 13032::rfp with an editing efficiency of $90.0 \%$ (Additional file 1: Figure S7). The achievable genome editing of different C. glutamicum strains with slight modification of

Table 1 CRISPR/Cas9-mediated gene deletion and insertion in C. glutamicum wild-type strains

\begin{tabular}{llcccc}
\hline Strain & Plasmids used & $\begin{array}{l}\text { Deleted fragment } \\
\text { size (bp) }\end{array}$ & $\begin{array}{l}\text { Inserted fragment } \\
\text { size (bp) }\end{array}$ & $\begin{array}{l}\text { Homologous arm } \\
\text { size (bp) }\end{array}$ & Efficiency \\
\hline ATCC 13869 & pCas9 and pgRNA3 & 664 & 0 & $\sim 1000$ & $30.8 \%(4 / 13) ; 35.7 \%(5 / 14) ;$ \\
& & & $78.3 \%(2 / 6)$ & $28.6 \%(4 / 14)$ \\
ATCC 13032 & pCas9 and pgRNA4 & 664 & 0 & $\sim 1000$ & $\sim 1000$ \\
& pCas9 and pgRNA3 & 664 & 0 & $\sim 500$ & $50.0 \%(2 / 4)$ \\
& pCas9 and pgRNA8 & 664 & 0 & $\sim 1000$ & $40.0 \%(2 / 5)$ \\
& pCas9 and pgRNA9 & 8083 & 781 & $\sim 1000$ & $62.5 \%(5 / 8)$ \\
& pCas9 and pgRNA4 & 664 & 3626 & $\sim 1000$ & $50.0 \%(4 / 8)$ \\
\hline
\end{tabular}


inducer usage suggests a possible broader applicability of the CRISPR/Cas9-mediated genome editing toolbox.

\section{Single-nucleotide editing}

Adaptive evolution and mutagenesis breeding usually generate hundreds of single nucleotide polymorphisms (SNPs) in the genome sequence. Single-nucleotide editing is a key enabling strategy to investigate the function of SNPs and assemble positive mutations (SNPs) to construct hyper producing strains. To explore the application of CRISPR/Cas9 system in single-nucleotide editing, a ssDNA ( $r f p$-off2, Additional file 1: Table S1) was designed to change $\mathrm{C}$ to $\mathrm{G}$ in $r f p$ gene (three bases upstream of the PAM sequence), which generated a stop codon. Similar editing efficiency (90.0\%) was obtained (Additional file 1: Figure S8), demonstrating that the CRISPR/Cas9 system could be applied to precise base editing.

\section{Double-locus editing}

Since ssDNA-directed recombineering achieved considerably high editing efficiency of single-locus editing, we then doubled the number of editing targets. $r f p$ and rps $L$ were selected as targets for double-locus editing and pgRNA7 that possessed two gRNA expression cassettes targeting $r f p$ and $r p s L$ were constructed (Fig. 5a). When C. glutamicum ATCC 13032::rfp harboring pgRNA7 was co-transformed with pCas9 and two kinds of ssDNAs (rfp-off1 and rpsL-K43R, Additional file 1: Table S1), only ten colonies were obtained, which were much fewer than those obtained in single-locus editing. All the ten colonies were verified and four of them were the expected double-mutants, demonstrating a double-locus editing efficiency of $40.0 \%$ (Fig. $5 \mathrm{~b}$ ). With the number of editing loci increased, the number of colonies obtained decreased dramatically. Therefore, simultaneous editing of more than two loci is still difficult to achieve. It is speculated that higher recombination efficiency would be helpful.

\section{Discussion}

As a gram-positive bacterium with good genomic stability, C. glutamicum is more difficult to engineer than genetically tractable hosts such as $E$. coli $[40,48]$. CRISPR/Cas9-mediated ssDNA recombineering was developed for deleting $400 \mathrm{bp}$ chromosomal fragment in C. glutamicum in the time this manuscript was being prepared [35]. However, gene deletion and insertion with plasmid-borne editing templates that are key enabling techniques for reconstruction and integration of metabolic pathways are still in demand. In this study, a tailormade CRISPR/Cas9 toolbox was developed for efficient and comprehensive engineering of C. glutamicum. Notably, gene deletion and insertion with plasmid-borne editing templates were efficiently implemented. Moreover, single-nucleotide editing and double-locus editing were achieved at efficiencies of 90.0 and $40.0 \%$, respectively, which will considerably accelerate precise genome editing of C. glutamicum.

S. pyogenes Cas9 is suggested to be toxic to C. glutamicum and difficult to be introduced into C. glutamicum $[35,36]$. As an alternative, F. novicida CRISPR/Cpf1 system was recruited to perform genetic manipulation of $C$. glutamicum recently [36]. Compared to the CRIPSR/Cpf1 tool, the present CRISPR/Cas9 tool has some distinct advantages. First, the efficiencies of CRISPR/Cas9-mediated gene deletion and insertion using plasmid-borne editing templates were higher than those obtained by CRIPSR/Cpf1. Second, the CRISPR/Cas9 system is more suitable for genome-wide scale engineering of C. glutamicum. Because Cpf1 utilizes a T-rich PAM and Cas9 utilizes NGG as PAM [36, 37, 49], Cas9 has considerably more editing targets in C. glutamicum. A bioinformatics analysis of the genome sequence of C. glutamicum ATCC 13032 revealed 332,289 Cas9 targets in coding sequences, which is $169.6 \%$ of the number of Cpf1 targets $(195,978)$ (Additional file 2: Table S2). Third, Cas9 possesses better targeting specificity and can be used for precise editing, including single-nucleotide editing. Since Cpf1 cannot distinguish a single-nucleotide mismatch in seed sequence, additional synonymous mutations need to be introduced near the targeting position [36, 37]. However, even a single synonymous mutation may alter the secondary structure of the mRNA and the expression of the mutant protein [50], limiting application of the CRIPSR/ Cpf1 tool in precise editing.

Considering the advantages of CRISPR/Cas9 system, attempts to introduce the $S$. pyogenes cas 9 gene with native codon into C. glutamicum were made but failed, which may be attributable to its strong expression and non-specific binding to the chromosomal DNAs [35, 36]. To reduce the translation efficiency, the cas 9 gene originally codon-optimized for atinomycetal genomes was recruited [35]. However, in this study, the cas 9 gene with native codon was successfully transformed into $C$. glutamicum and used for counter-selection. The same IPTG-inducible promoter $P_{t a c}$ but different ribosome binding sites from the previous studies were used here, which may lead to successful introduction of the cas 9 gene. The previous and present results all suggest that fine-tuning of Cas9 expression is crucial for application of CRISPR/Cas9 system in bacteria. Another unexpected crucial factor was discovered that the frequently used gRNA terminator derived from $S$. pyogenes $\left(T_{S p}\right)$ did not terminate transcription in C. glutamicum. As a consequence, redundant RNA segments would be transcribed with the gRNA, which might affect the function 
a

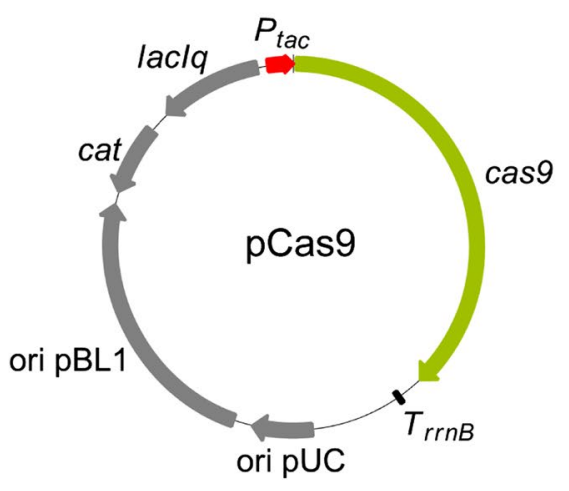

b Colony

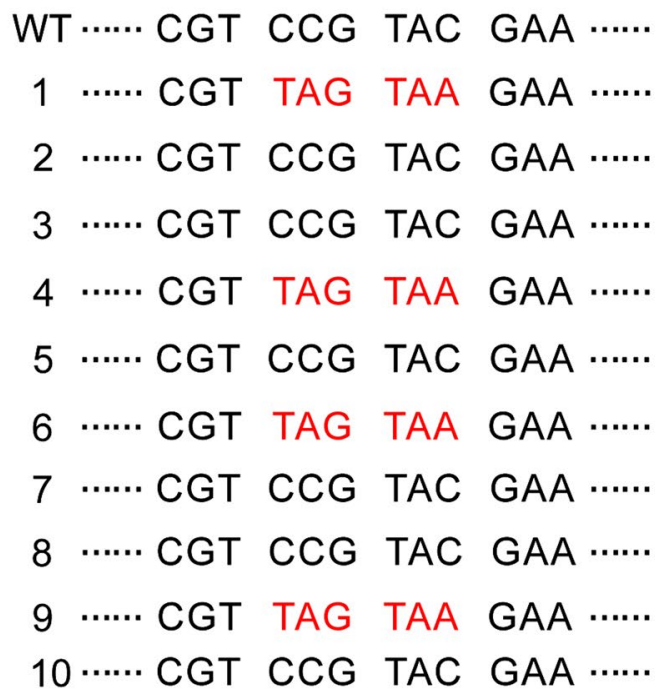

rfp gene

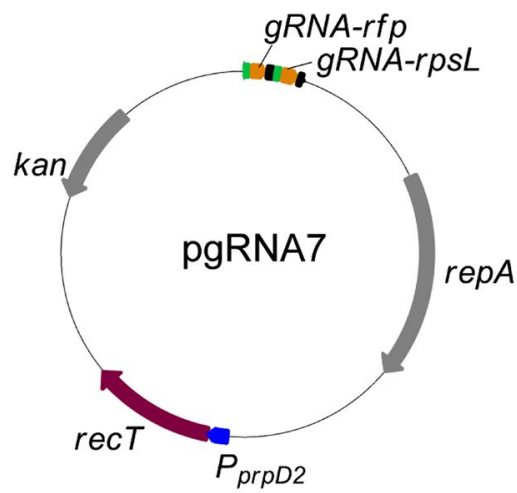

rpsL gene

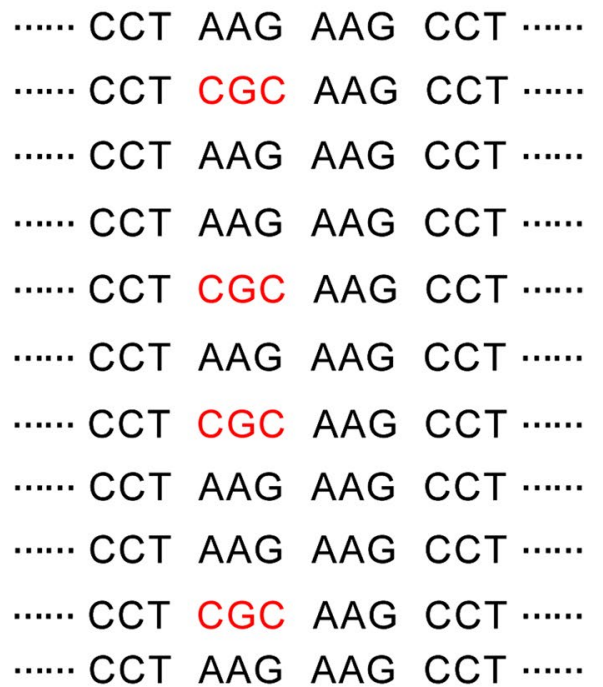

Fig. 5 Double-locus editing in in C. glutamicum. a Schematic representation of pCas9 and pgRNA7 which were used for ssDNA-directed recombineering. b Mutants verification by sequencing of $r f p$ and $r p s L$ genes. Plasmid pgRNA7 that simultaneously expressed two gRNAs targeting $r f p$ and rpsL was first transformed into C. glutamicum ATCC 13032::rfp. The resultant strain was then induced to express RecT and co-transformed with pCas9 and two kinds of synthetic ssDNAs (10 $\mu \mathrm{g} \mathrm{rfp-off1}$ and $10 \mu \mathrm{g} r p s L-K 43 R$, Additional file 1: Table S1). After recovery, cells were spread on SGY plates supplemented with $\mathrm{Km}, \mathrm{Cm}$, and IPTG $(0.01 \mathrm{mM})$ to induce Cas9 expression for counter-selection. Colonies were picked and verified by gene sequencing

of Cas9-gRNA complex. Deletion of the 185 bp redundant segment between $T_{S p}$ and plasmid-borne $T_{r r n B}$ resulted in an acceptable escape rate $\left(1.3 \pm 0.2 \times 10^{-2}\right)$ for counter-selection.

During CRISPR/Cas9-mediated microbial genome editing progress, production of false positives seemed inevitable even in $E$. coli, of which the escape rate of counter-selection was quite low [11, 12, 16]. CRISPR/ Cpf1-assisted editing also suffers from the same problems [36]. Although such false positives will disturb screening of edited cells, the mechanism behind the phenomenon remained unclear [16]. Here we demonstrated that modification on the cas 9 gene was the main cause of producing such false positives (Fig. 2c). In the case of CRISPR/Cpf1-assisted editing, Cpf1 mutations may also be the major reason for false positives formation since editing efficiency decreased when continuous editing was conducted using the Cpf1 expression plasmid transformed in the former round of editing [36]. By using a one-step electrotransformation strategy to reduce replication of pCas9 in the host, we alleviated the problem and increased gene deletion efficiency significantly. Previous studies suggested that small amount of Cas 9 proteins produced by leaky expression can already lead to introduction of DSB resulting in SOS induction [51]. Our experiments also showed that even in the absence of gRNA, Cas9 expression hindered cell growth of $C$. glutamicum. Therefore, we hypothesized that such SOS 
induction in C. glutamicum may lead to modification of the cas 9 expression cassette. To overcome this obstacle, more strictly controlled promoter is needed for Cas9 and Cpf1 expression.

Electrotransformation and homologous recombination efficiencies of C. glutamicum are another two key factors that affects the acquisition of edited mutants [35]. In this study, C. glutamicum SL4, a derivative of strain ATCC 13869, was first selected as the target strain because of its high electrotransformation efficiency. Comparative genome analysis between strains SL4 and ATCC 13869 revealed some mutations in cell wall synthesis-related genes, such as flippase, arabinosyltransferase, and murein biosynthesis protein encoding genes. It is speculated that these mutations may contribute to the high electroporation efficiency of strain SL4 and further studies on the mechanism are underway. In some cases (e.g., gene deletion in $C$. beijerinckii), serial subculturing was applied to compensate for the low homologous recombination efficiency and enrich edited cells [26]. However, because false positives would outgrow edited cells, such strategy was unsuitable for the present case. Previous studies demonstrated that introducing exogenous recombinase or exonuclease benefited homologous recombination in C. glutamicum [35, 42, 52], which gave us inspiration to improve the editing efficiency of the CRISPR/Cas9 toolbox.

\section{Conclusions}

In summary, a CRISPR/Cas9 toolbox was developed for efficient and comprehensive engineering of the industrial workhorse C. glutamicum. Markerless gene deletion, gene insertion, single-nucleotide editing and doublelocus editing were achieved by using a customized twoplasmid-based CRISPR/Cas9 system and a simplified co-transformation strategy. This toolbox works well in several C. glutamicum strains and holds promise for renovating the genome editing of corynebacteria.

\section{Methods}

Microorganisms, cultivation conditions, and plasmids

All bacterial strains used in this study are listed in Table 2. E. coli $\mathrm{DH} 5 \alpha$ was used for general cloning and cultivated aerobically at $37{ }^{\circ} \mathrm{C}$ in Luria-Bertani (LB) broth. $\mathrm{Km}(25 \mu \mathrm{g} / \mathrm{mL})$ or $\mathrm{Cm}(20 \mu \mathrm{g} / \mathrm{mL})$ was added to LB broth as required. C. glutamicum ATCC 13869 and C. glutamicum ATCC 13032 were obtained from the American Type Culture Collection (ATCC). C. glutamicum SL4 was a derivative of strain ATCC 13869 with high electrotransformation efficiency. C. glutamicum SL4 and its derivatives were cultivated aerobically at $30^{\circ} \mathrm{C}$ in SGY medium containing $18 \mathrm{~g} / \mathrm{L}$ soya peptone, $5 \mathrm{~g} / \mathrm{L}$ glucose, $10 \mathrm{~g} / \mathrm{L}$ yeast extract, $1 \mathrm{~g} / \mathrm{L} \mathrm{K}_{2} \mathrm{HPO}_{4} \cdot 3 \mathrm{H}_{2} \mathrm{O}, 1 \mathrm{~g} / \mathrm{L}$ urea,
$0.5 \mathrm{~g} / \mathrm{L}$ succinic acid, and $10 \mu \mathrm{g} / \mathrm{L}$ biotin. C. glutamicum ATCC 13032, C. glutamicum ATCC 13869 and their derivatives were cultivated aerobically at $30{ }^{\circ} \mathrm{C}$ in SGY medium or LBHIS medium [53]. $\mathrm{Km}(25 \mu \mathrm{g} / \mathrm{mL}), \mathrm{Cm}$ $(5 \mu \mathrm{g} / \mathrm{mL})$, streptomycin (Str, $25 \mu \mathrm{g} / \mathrm{mL})$ or IPTG $(0.01$ to $1 \mathrm{mM}$ ) was added in the medium as required. All plasmids used in this study (Table 2) were constructed using ClonExpress II One Step Cloning Kit or ClonExpress MultiS One Step Cloning Kit (Vazyme, Nanjing, China), which facilitated ligation of two or more DNA fragments through 15-20 bp overlaps. Primers and synthetic ssDNAs used for genetic manipulation are listed in Additional file 1: Table S1.

\section{Electrotransformation protocol of C. glutamicum}

C. glutamicum SL4 and its derivatives cultivated overnight in SGY medium were inoculated into $100 \mathrm{~mL}$ fresh $\mathrm{SGY}$ medium to an $\mathrm{OD}_{600 \mathrm{~nm}}$ value of 0.3 . When $\mathrm{OD}_{600 \mathrm{~nm}}$ value reached $\sim 0.8$, cells were collected by centrifugation at $8000 \mathrm{rpm}$ and $4{ }^{\circ} \mathrm{C}$ for $10 \mathrm{~min}$. After washed with ice-cold deionized distilled water for 4 times, cells were resuspended in $0.5 \mathrm{~mL} 10.0 \%(\mathrm{v} / \mathrm{v})$ glycerol and $100 \mu \mathrm{L}$ aliquots of competent cells were obtained. DNA (less than $10 \mu \mathrm{L}$ ) was added to the competence cells and transferred to a $2 \mathrm{~mm}$ electroporation cuvette (BioRad) with parameters set at $2500 \mathrm{~V}$ and $5 \mathrm{~ms}$. Electroporations were performed with Eppendorf Electroporator 2510. After electroporation, $1 \mathrm{~mL}$ SGY medium was added immediately. Cells were incubated for $2-6 \mathrm{~h}$ at $30^{\circ} \mathrm{C}$, and spread on SGY plates supplemented with antibiotics and IPTG as required. The plates were incubated at $30{ }^{\circ} \mathrm{C}$ until colonies appeared. Regarding to C. glutamicum ATCC 13869, C. glutamicum ATCC 13032 and their derivatives, preparation of competent cells and electrotransformation were performed according to the protocols described previously [53].

\section{Construction of Cas9 and gRNA expression plasmids and determination of Cas9-induced lethality}

To optimize the promoter for Cas9 expression, plasmids pRfp1 and pRfp2 were constructed. The first $180 \mathrm{bp}$ of cas 9 gene and the full-length $r f p$ gene were amplified by PCR from pRed_Cas9_recA_Lpoxb300 [54] and pECXK99E-rfp using primer pairs cas $9^{180 \mathrm{bp}_{-}} 1 /$ cas $^{180 \mathrm{~b}} 9_{-2}$ and $r f p-1 / r f p-2$, respectively. The cas $9^{180 \mathrm{bp}}$ segment and $r f p$ gene were subcloned into the HindIII and PstI sites of pXMJ19 under control of $P_{t a c}$, producing pRfp1. $P_{p r p D 2}$, cas $9^{180 \mathrm{bp}}, r f p$, and pXMJ19 backbone were amplified by PCR using primer pairs $P_{p r p D 2}-1 / P_{p r p D 2}-2$, cas $9^{180 \mathrm{bp}}$ $3 /$ cas $9^{180 b \mathrm{~b}}-4, \quad r f p-1 / r f p-2$, and pXMJ19-1/pXMJ19-2, respectively, and assembled into $\mathrm{pRfp} 2$. pRfp1 and pRfp2 were introduced into C. glutamicum SL4 through electrotransformation separately. The transformants were 
Table 2 Strains and plasmids used in this study

\begin{tabular}{|c|c|c|}
\hline Strain or plasmid & Description $^{a}$ & Reference or source \\
\hline \multicolumn{3}{|l|}{ E. coli } \\
\hline $\mathrm{DH} 5 \mathrm{a}$ & General cloning host & TaKaRa \\
\hline MG1655 & Source of rect & Lab collection \\
\hline \multicolumn{3}{|l|}{ C. glutamicum } \\
\hline ATCC 13869 & Wild-type strain & ATCC \\
\hline ATCC 13032 & Wild-type strain & ATCC \\
\hline ATCC 13032::rfp & ATCC 13032 derivative with insertion of a rfp cassette & Lab collection \\
\hline SL4 & ATCC 13869 derivative with high electrotransformation efficiency & Lab collection \\
\hline SL4 (pCas9) & SL4 derivative harboring pCas 9 & This work \\
\hline $\mathrm{SL} 4 \triangle / d h A$ & SL4 derivative with IdhA deletion & This work \\
\hline $\mathrm{SL} 4 \Delta / d h A:: r f p$ & SL4 derivative with insertion of a $r f p$ cassette in $I d h A$ & This work \\
\hline $\mathrm{SL} 4 \Delta / d h A:: r f p(p g R N A 5)$ & SL4 $\triangle$ /dhA::rfp derivative harboring pgRNA5 & This work \\
\hline $\mathrm{SL} 4 \Delta / d h A:: . r f p^{\text {off1 }}$ & $\begin{array}{l}\text { SL } 4 \triangle / d h A:: \text { ffp derivative with nonsense mutation in } r f p \text {, constructed by ssDNA (rfp-off } 1 \text { ) } \\
\text { recombineering }\end{array}$ & This work \\
\hline SL4 (pgRNA6) & SL4 derivative harboring pgRNA6 & This work \\
\hline SL4 rpsL ${ }^{K 43 R}$ & SL4 derivative with K43R mutation of rps $L$ & This work \\
\hline SL4 (pcas9 + pgRNA1) & SL4 derivative harboring pcas 9 and pgRNA1 & This work \\
\hline SL4 (pcas9 + pgRNA2) & SL4 derivative harboring pcas 9 and pgRNA2 & This work \\
\hline ATCC $13869 \Delta / d h A$ & ATCC 13869 derivative with IdhA deletion & This work \\
\hline ATCC $13032 \Delta / d h A$ & ATCC 13032 derivative with IdhA deletion & This work \\
\hline ATCC $13032 \triangle \mathrm{cg} / 1776-\mathrm{cg} / 178$ & ATCC 13032 derivative with cg/1776-cg/178 deletion & This work \\
\hline ATCC 13032::/acZ & ATCC 13032 derivative with lacZ insertion & This work \\
\hline ATCC 13032::rfp (pgRNA5) & ATCC 13032::rfp derivative harboring pgRNA5 & This work \\
\hline ATCC 13032::rfp off1 & $\begin{array}{l}\text { ATCC 13032::rfp derivative with nonsense mutation in rfp, constructed by ssDNA (rfp-off1) } \\
\text { recombineering }\end{array}$ & This work \\
\hline ATCC 13032::rff off2 & $\begin{array}{l}\text { ATCC 13032::rfp derivative with nonsense mutation in rfp, constructed by ssDNA (rfp-off2) } \\
\text { recombineering }\end{array}$ & This work \\
\hline \multicolumn{3}{|l|}{ Plasmid } \\
\hline pEC-XK99E & Expression vector of $C$. glutamicum, IPTG-inducible promoter $P_{t r c} \mathrm{Km}^{R}$ & {$[55]$} \\
\hline pXMJ19 & Expression vector of C. glutamicum, IPTG-inducible promoter $P_{\text {tac }} \mathrm{Cm}^{\mathrm{R}}$ & {$[56]$} \\
\hline pRed_Cas9_recA_Apoxb300 & Source of cas 9 gene and gRNA fragment & {$[54]$} \\
\hline pEC-XK99E-rfp & Source of $r f p$ gene & Lab collection \\
\hline pCas9 & pXMJ19 derivative carrying cas 9 gene, driven by IPTG-inducible promoter $P_{\text {tac }}$ & This work \\
\hline pRfp1 & pXMJ19 derivative carrying cas9 $9^{180 \mathrm{bp}}$-rfp artificial gene, driven by IPTG-inducible promoter $P_{\text {tac }}$ & This work \\
\hline pRfp2 & 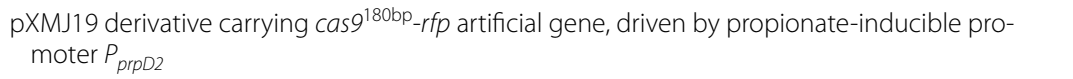 & This work \\
\hline pRfp3 & pEC-XK99E derivative carrying gRNA-rfp cassette, driven by constitutive promoter $P_{11 F}$ & This work \\
\hline pRfp4 & $\begin{array}{l}\text { pEC-XK99E derivative carrying gRNA (with } T_{s p} \text { deleted)-rfp cassette, driven by constitutive } \\
\text { promoter } P_{11 F}\end{array}$ & This work \\
\hline pgRNA1 & pEC-XK99E derivative carrying gRNA targeting IdhA, driven by IPTG-inducible promoter $P_{\text {tac }}$ & This work \\
\hline pgRNA2 & $\begin{array}{l}\text { pEC-XK99E derivative carrying gRNA targeting /dhA, driven by constitutive promoter } P_{11 F} \text {, fol- } \\
\text { lowed by } T_{r m B} \text { without the } 185 \text { bp redundant segment }\end{array}$ & This work \\
\hline pgRNA3 & $\begin{array}{l}\text { pgRNA2 derivative carrying homologous arms ( 1 kb) for IdhA deletion and corresponding } \\
\text { gRNA cassette }\end{array}$ & This work \\
\hline pgRNA4 & $\begin{array}{l}\text { pgRNA3 derivative with a rfp cassette inserted between the two homologous }(\sim 1 \mathrm{~kb}) \text { arms for } \\
\text { replacing } / \mathrm{dhA} \text { with the } r f p \text { cassette }\end{array}$ & This work \\
\hline pgRNA5 & $\begin{array}{l}\text { pgRNA2 derivative carrying gRNA targeting } r f p \text { and a } P_{p r p D 2}-r e c T \text { cassette for ssDNA-directed } r f p \\
\text { editing }\end{array}$ & This work \\
\hline pgRNA6 & $\begin{array}{l}\text { pgRNA5 derivative carrying gRNA targeting } r p s L \text { and a } P_{p r p D 2}{ }^{-r e c T} \text { cassette for ssDNA-directed } \\
\text { rps } L \text { editing }\end{array}$ & This work \\
\hline pgRNA7 & $\begin{array}{l}\text { pgRNA5 derivative carrying two gRNAs targeting } r f p \text { and } r p s L \text { and a } P_{\text {prpD2 }} \text {-recT cassette for } \\
\text { double-locus editing }\end{array}$ & This work \\
\hline
\end{tabular}


Table 2 continued

\begin{tabular}{llc}
\hline Strain or plasmid & Description $^{\text {a }}$ & Reference or source \\
\hline pgRNA8 & pgRNA3 derivative carrying homologous arms $(\sim 0.5 \mathrm{~kb})$ for ldhA deletion and corresponding & This work \\
& gRNA cassette & \\
pgRNA9 & anNA3 derivative carrying homologous arms $(\sim 1 \mathrm{~kb})$ for cgl1776-cg/1781 deletion (8083 bp) & This work \\
pgRNA10 & $\begin{array}{l}\text { pgRNA3 derivative carrying homologous arms }(\sim 1 \mathrm{~kb}), \text { lac } Z \text { cassette }(3626 \mathrm{bp}) \text { and correspond- } \\
\text { ing gRNA cassette for lac } Z \text { cassette insertion }\end{array}$ & This work \\
\hline
\end{tabular}

${ }^{a} \mathrm{Km}^{\mathrm{R}}$ and $\mathrm{Cm}^{\mathrm{R}}$ represent resistance to kanamycin and chloramphenicol, respectively

cultivated in the presence or absence of $1 \mathrm{mM}$ IPTG (for $\mathrm{pRfp} 1$ ) or $1 \mathrm{~g} / \mathrm{L}$ sodium propionate (for $\mathrm{pRfp} 2$ ). Cells of the stationary growth phase were used to detect their fluorescence outputs using a microplate reader $(\lambda$ excitation $=560 \mathrm{~nm}, \lambda$ emission $=607 \mathrm{~nm}$ ).

To construct plasmid pCas9, the cas 9 gene was amplified by PCR using primer pair cas $9-1 /$ cas $9-2$ from pRed Cas9_recA_Lpoxb300 [54] and inserted into the HindIII and Pst I sites of pXMJ19 under control of $P_{t a c}$. Plasmid pgRNA1 was produced by ligating lacIq- $P_{t a c}$ fragment, gRNA fragment targeting $l d h A$ and pEC-XK99E backbone. The three DNA fragments were amplified by PCR from pXMJ19, pRed_Cas9_recA__poxb300 and pECXK99E using primer pairs $P_{t a c}-1 / P_{t a c}-2$, gRNA-3/gRNA-4 and pEC-XK99E-4/pEC-XK99E-5, respectively. The gRNA targeting ldhA contained a base-pairing region (N24 5'-GTGGATATCCTGACCTACGCAGTG-3'), a Cas9 handle and a S. pyogenes terminator $\left(T_{s p}\right)$. In plasmid pgRNA1, the $r r n B$ terminator $\left(T_{r r n B}\right)$ from pEC-XK99E backbone located in the185 bp downstream of $T_{s p}$.

In order to confirm whether $T_{s p}$ can work in C. glutamicum, plasmids pRfp3 and pRfp4 were constructed. pRfp3 was a derivative of pEC-XK99E carrying the gRNA (targeting $l d h A$ ) and $r f p$ gene driven by the constitutive promoter $P_{11 F^{*}} . P_{11 F}$-gRNA was amplified by PCR from pgRNA1 using primer pair gRNA-1/gRNA-2. The $r f p$ gene was amplified by PCR from pEC-XK99E-rfp using primer pair $r f p-4 / r f p-5$, and the pEC-XK99E backbone was amplified using primer pair pEC-XK99E-1/pECXK99E-2. To construct pRfp4, the backbone of pRfp3 was amplified by PCR to remove the $T_{s p}$ and $r f p$ gene using primer pair pEC-XK99E-3/pEC-XK99E-1. The $r f p$ gene was amplified by PCR from pRfp3 using primer pair $r f p-5 / r f p-6$ and ligated to the backbone of pRfp3, resulting in pRfp4. pRfp3 and pRfp4 were introduced into E. coli MG1655 and C. glutamicum SL4 through electrotransformation separately. Cells of the stationary growth phase were used to detect their fluorescence outputs using a microplate reader $(\lambda$ excitation $=560 \mathrm{~nm}, \lambda$ emission $=607 \mathrm{~nm}$ ).

Plasmid pgRNA2 was derived from pgRNA1 by replacing $P_{t a c}$ with $P_{11 F}$ and deleting the $185 \mathrm{bp}$ region between
$T_{s p}$ and $T_{r r n B}$. The $P_{11 F^{-}}$gRNA fragment was amplified by PCR from pgRNA1 using primer pair gRNA-1/gRNA-6. pgRNA1 backbone was amplified by PCR using primer pair pEC-XK99E-6/pEC-XK99E-2 to remove the $185 \mathrm{bp}$ region. The two fragments were ligated to form pgRNA2.

To determine the escape rate of counter-selection, $1 \mu \mathrm{g}$ pCas9 was transformed into C. glutamicum SL4. Cells were spread on SGY plates supplemented with $\mathrm{Cm}$ after $2 \mathrm{~h}$ recovery. After cultivated at $30{ }^{\circ} \mathrm{C}$ for 2 days, colonies were picked and verified by colony PCR. The correct transformant was designated as C. glutamicum SL4 (pCas9). Then pgRNA1 and pgRNA2 were transformed into C. glutamicum SL4 (pCas9) separately. Correct transformants were cultivated, diluted and spread on SGY plates containing $\mathrm{Km}$ and $\mathrm{Cm}$, with or without IPTG $(1 \mathrm{mM})$. The escape rate of counter-selection was calculated by colony counting.

\section{Gene deletion and insertion using CRISPR/Cas9}

To provide editing template for $l d h A$ deletion, two homologous arms of $l d h A$ ( $\sim 1 \mathrm{~kb}$ for each arm) were amplified by PCR from the genomic DNA of C. glutamicum SL4 using primer pairs $l d h A-1 / l d h A-2$ and ldhA-3/ldhA-4, respectively. The backbone of pgRNA2 was amplified by PCR using primer pair pEC-XK99E-8/ pEC-XK99E-9. pgRNA3 was constructed by ligating the two homologous arms and pgRNA2 backbone. Plasmid pgRNA4 was derived from pgRNA3 by inserting a $r f p$ cassette between the two homologous arms of $l d h A$. The $r f p$ cassette and pgRNA3 backbone were amplified by PCR using primer pairs $r f p-7 / r f p-8$ and $l d h A-2 / l d h A-5$, respectively. The two fragments were ligated to form pgRNA4. Plasmid pgRNA8 was derived from pgRNA3 by replacing the $\sim 1 \mathrm{~kb}$ homologous arms with $\sim 500 \mathrm{bp}$ homologous arms for $l d h A$ deletion. Primer pairs $l d h A$ 6/ldhA-7 and pEC-XK99E-8/pEC-XK99E-9 were used to amplify the homologous arms and plasmid backbone from plasmid pgRNA3. The two fragments were ligated to form pgRNA8. Plasmid pgRNA9 was constructed to delete the cgl1776-cgl1781 fragment (8083 bp). Primer pairs pEC-XK99E-13/pEC-XK99E-8 and pEC-XK99E-9/ pEC-XK99E-14 were used to amplify two plasmid 
backbone fragments from plasmid pgRNA3. Primer pairs 8083-1/8083-2 and 8083-3/8083-4 were used to amplify two homologous arm fragments from the genomic DNA of C. glutamicum ATCC 13032. The four fragments were ligated to form pgRNA9. Plasmid pgRNA10 was constructed to insert the lac $Z$ cassette (3626 bp) into the genomic locus between $\mathrm{cgl0900}$ and $\mathrm{cgl0901}$ in C. glutamicum ATCC 13032. Primer pairs pEC-XK99E-15/ pEC-XK99E-8 and pEC-XK99E-9/pEC-XK99E-16 were used to amplify two plasmid backbone fragments from plasmid pgRNA3. Primer pairs 3626-1/3626-2 and 36263/3626-4 were used to amplify two homologous arm fragments from the genomic DNA of C. glutamicum ATCC 13032. Primer pair lacZ-1/lacZ-2 was used to amplify the lac $Z$ cassette from the genomic DNA of E. coli MG1655. The five fragments were ligated to form pgRNA10.

Regarding to ldhA deletion in C. glutamicum SL4, $1 \mu \mathrm{g}$ pgRNA3 was transformed into C. glutamicum SL4 (pCas9). Cells were recovered at $30{ }^{\circ} \mathrm{C}$ for $6 \mathrm{~h}$ and then spread on SGY plates supplemented with $\mathrm{Km}, \mathrm{Cm}$, and IPTG $(1 \mathrm{mM})$. Plates were incubated at $30{ }^{\circ} \mathrm{C}$ for $2-3$ days until colonies appeared. The C. glutamicum SL4 $\Delta l d h A$ mutants were verified by colony PCR using primer pair ldhA-up/ldhA-down. PCR products of edited cells were $1604 \mathrm{bp}$, and those of wild-type cells were $2268 \mathrm{bp}$. When the one-step electrotransformation strategy was used, $3 \mu \mathrm{g}$ pCas9 and $1 \mu \mathrm{g}$ pgRNA3 were co-transformed into C. glutamicum SL4. Mutants screening and verification were performed using procedures described above. Regarding to $r f p$ insertion in C. glutamicum SL4, the one-step electrotransformation strategy described above was used except that pCas9 and pgRNA4 were co-transformed into C. glutamicum SL4 and primer pair $r f p$-up/ldhA-down was used for PCR verification of $C$. glutamicum SL4 $\Delta l d h A:: r f p$ mutants. PCR products of edited cells were $1449 \mathrm{bp}$.

For gene deletion and insertion in C. glutamicum ATCC 13869 and ATCC 13032, pCas9 and pgRNA3 (pgRNA4, pgRNA8, pgRNA9, or pgRNA10) were cotransformed. After electroporation, $1 \mathrm{~mL}$ LBHIS broth was added immediately and the suspension was quickly incubated for $6 \mathrm{~min}$ at $46^{\circ} \mathrm{C}$ [53]. Cells were then incubated for $6 \mathrm{~h}$ at $30^{\circ} \mathrm{C}$ and subsequently spread on SGY or LBHIS plates supplemented with $\mathrm{Km}, \mathrm{Cm}$, and IPTG (0.01 or $0.1 \mathrm{mM}$ ), Plates were incubated at $30{ }^{\circ} \mathrm{C}$ for 2-3 days until colonies appeared. Mutants verification were performed by colony PCR using the primer pairs shown in Additional file 1: Table S1.

\section{CRISPR/Cas9-mediated ssDNA recombineering}

Plasmid pgRNA5 was construct by inserting a $P_{p r p D 2}-r e c T$ cassette into pgRNA2 and replacing the base-pairing region of gRNA targeting ldhA with a base-pairing region targeting $r f p$ (N20, 5'-GCGGTCTGGGTACCTTCGTA-3'). A part of pgRNA2 backbone was amplified by PCR using primer pair gRNA-7/ pEC-XK99E-8. N20 for $r f p$ was added to the backbone by primer gRNA-7. A second part of pgRNA2 backbone was amplified by PCR using primer pair pEC-XK99E-9/pECXK99E-10. $P_{p r p D 2}$ and $r e c T$ were amplified by PCR using primer pairs $P_{p r p D 2}-3 / P_{p r p D 2}-4$ from the genomic DNA of C. glutamicum ATCC 13032 and recT-1/recT-2 from the genomic DNA of E. coli MG1655, respectively. The four fragments were ligated to form the pgRNA5. To construct plasmid pgRNA6, the backbone of pgRNA5 was amplified by PCR using primer pair pEC-XK99E-11/pECXK99E-12 with replacement of the base-pairing region of gRNA targeting $r f p$ with a base-pairing region targeting rpsL (N20, 5'-AGAGCAGAGTTAGGCTTCTT-3'). Then the fragment was ligated upon itself to form the pgRNA6.

CRISPR/Cas9-mediated ssDNA recombineering was conducted using a two-step electrotransformation strategy. Firstly, $1 \mu \mathrm{g}$ pgRNA5 was transformed into $C$. glutamicum SL4 $\Delta l d h A:: r f p$. The competent cells of $C$. glutamicum SL4AldhA::rfp (pgRNA5) were prepared by addition of $1 \mathrm{~g} / \mathrm{L}$ sodium propionate to induce RecT recombinases expression, and then co-transformed with 1-2 $\mu \mathrm{g}$ pCas9 and 6-15 $\mu \mathrm{g}$ synthetic ssDNA ( $r f p$-off1 or $r f p$-off2, Additional file 1: Table S1). Cells were recovered at $30{ }^{\circ} \mathrm{C}$ for $6 \mathrm{~h}$ and then spread on SGY plates supplemented with $\mathrm{Km}, \mathrm{Cm}$, and IPTG $(1 \mathrm{mM})$. Plates were incubated at $30{ }^{\circ} \mathrm{C}$ for $2-3$ days until colonies appeared. Recombination events were verified by detecting the fluorescence outputs of colonies. C. glutamicum SL4 $\Delta l d h A:: r f p^{\text {off1 }}$ mutants were further verified by gene sequencing. When rps $L$ was selected as a target of ssDNA recombineering, similar procedures described above were used with small modifications. Plasmid pgRNA6 was first transformed into C. glutamicum SL4. The competent cells of C. glutamicum SL4 (pgRNA6) was then transformed with pCas9 and synthetic ssDNA (rpsLK43R, Additional file 1: Table S1). C. glutamicum SL4 $r p s L^{\mathrm{K} 43 \mathrm{R}}$ mutants were verified by $\operatorname{Str}^{\mathrm{R}}$ phenotype test and gene sequencing. To perform ssDNA recombineering in C. glutamicum ATCC 13032, an engineered strain (ATCC 13032::rfp) was used and $r f p$ was selected as a target. The procedures were similar with those for recombineering C. glutamicum SL4 $\Delta l d h A:: r f p$ except that $0.01 \mathrm{mM}$ IPTG was used for inducing Cas9 expression.

\section{Double-locus editing}

$r f p$ and $r p s L$ were selected as targets for double-locus editing in C. glutamicum ATCC 13032::rfp. Plasmid pgRNA7 that simultaneously expressed two gRNAs targeting $r f p$ and $r p s L$ was constructed by inserting a gRNA fragment targeting rpsL into pgRNA5. The 
gRNA fragment targeting rpsL was amplified by PCR using primer pair gRNA-8/gRNA-9 from pgRNA6. The pgRNA5 backbone was amplified by PCR using primer pair gRNA-10/pEC-XK99E-2. The two fragments were ligated to form pgRNA7. The procedures were similar with those for single-gene editing in C. glutamicum ATCC 13032 by using CRISPR/Cas9-mediated ssDNA recombineering, except that two kinds of synthetic ssDNAs $(10 \mu \mathrm{g} r f p$-off1 and $10 \mu \mathrm{g} r p s L-K 43 R$, Additional file 1: Table S1) were transformed with pCas9 simultaneously.

\section{Plasmid curing}

To remove Cas9 and gRNA expression plasmids from edited cells, the edited colony was inoculated into $100 \mathrm{~mL}$ of SGY medium without antibiotics and cultivated for $12 \mathrm{~h}$. The culture was serially transferred into fresh medium with an inoculum size of $0.1 \%(\mathrm{v} / \mathrm{v})$ for another two times, diluted, and spread on SGY plates without antibiotics. Colonies were confirmed as cured by determining their sensitivity to $\mathrm{Km}$ and $\mathrm{Cm}$.

\section{Additional files}

Additional file 1: Figure S1. PCR verification of IdhA deletion using CRISPR/Cas9 and the two-step electrotransformation strategy in Corynebacterium glutamicum SL4. M, DNA marker; -, wild-type control; 1-20, twenty colonies. pCas9 was firstly transformed into C. glutamicum SL4. pgRNA3 was then transformed into strain SL4 (pCas9) and cells were spread on SGY plates supplemented with $\mathrm{Km}, \mathrm{Cm}$ and IPTG $(1 \mathrm{mM})$ immediately after recovery. After cultivated at $30^{\circ} \mathrm{C}$, twenty small colonies instead of the abnormally large colonies were picked to perform colony PCR using a pair of primers IdhA-up and IdhA-down. Figure S2. $P C R$ verification of IdhA deletion using CRISPR/Cas9 and the one-step electrotransformation strategy in C. glutamicum SL4. (a) Replicate 2. M, DNA marker; -, wild-type control; 1-6, six colonies. (b) Replicate 3. M, DNA marker; -, wild-type control; 1-9, nine colonies. pCas9 and pgRNA3 were co-transformed into C. glutamicum SL4 simultaneously and cells were spread on SGY plates supplemented with $\mathrm{Km}, \mathrm{Cm}$ and IPTG (1 mM) immediately after recovery. After cultivated at $30^{\circ} \mathrm{C}$, all the small colonies on the plates were picked to perform colony $\mathrm{PCR}$ using a pair of primers IdhA-up and IdhA-down. Figure S3. PCR verification of IdhA deletion using pCas9 and pgRNA3-derivative plasmid that harbored no targeting spacer in C. glutamicum SL4. M, DNA marker; -, wild-type control; 1-20, twenty colonies. pCas9 and pgRNA3-derivative plasmid that harbored no targeting spacer were co-transformed into C. glutamicum SL4 simultaneously and cells were spread on SGY plates supplemented with $\mathrm{Km}, \mathrm{Cm}$ and IPTG (1 mM) immediately after recovery. After cultivated at $30^{\circ} \mathrm{C}$, twenty colonies on the plates were picked to perform colony PCR using a pair of primers IdhA-up and IdhA-down. Figure S4. PCR verification of rfp insertion using CRISPR/Cas9 and plasmid-borne editing template in C. glutamicum SL4. (a) Replicate 2. M, DNA marker; -, wild-type control; 1-13, thirteen colonies. (b) Replicate 3. M, DNA marker; -, wild-type control; 1-9, nine colonies. pCas9 and pgRNA4 were co-transformed into C. glutamicum SL4 simultaneously and cells were spread on SGY plates supplemented with $\mathrm{Km}, \mathrm{Cm}$ and IPTG (1 mM) immediately after recovery. After cultivated at $30^{\circ} \mathrm{C}$, all the small colonies on the plates were picked to perform colony PCR using a pair of primers rfp-up and IdhA-down.

Figure S5. Transformants harboring pCas9, pgRNA5, and ssDNA (rfp-off1). Plasmid pgRNA5 was first transformed into C. glutamicum SL4 $\triangle$ /dhA::rfp. The resultant strain SL4 $\triangle / d h A:: . f p$ (pgRNA5) was cultivated with addition of propionate to induce RecT expression, and then electrocompetent cells were prepared. Next, cells were transformed with pCas 9 and ssDNA (rfp-off1) and spread on SGY plates supplemented with $\mathrm{Km}, \mathrm{Cm}$ and IPTG (1 mM) immediately after recovery. Figure S6. Fluorescence output detection of candidate mutants of C. glutamicum SL4 $\triangle / d h A:$ :rfp ${ }^{\text {off } 1}$. (a) Replicate 2. -, wild-type C. glutamicum SL4 control; +, C. glutamicum SL4 $\triangle l d h A:$ :rfp; 1-10, ten colonies. (b) Replicate 3. -, wild-type C. glutamicum SL4 control; +, C. glutamicum SL4 $\triangle$ IdhA::rfp; 1-10, ten colonies. Figure S7. Fluorescence output detection of candidate mutants of $C$. glutamicum ATCC 13032:::rfp off1. --, wild-type C. glutamicum ATCC 13032 control; +, C. glutamicum ATCC 13032::rfp; 1-10, ten colonies. Figure S8. Fluorescence output detection of candidate mutants of C. glutamicum

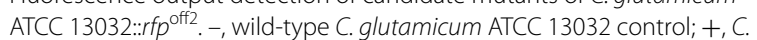
glutamicum ATCC 13032::rfp; 1-10, ten colonies. Table S1. Sequences of primers and ssDNAs used in this study.

Additional file 2: Table S2. Analysis of PAMs for Cas9 and Cpf1 in the genome sequence of C. glutamicum ATCC 13032.

\section{Abbreviations}

SsDNA: single-strand DNA; FACS: fluorescence-activated cell sorting; CRISPR: clustered regularly interspaced short palindromic repeats; Cas: CRISPR-associated proteins; dCas9: nuclease-deactivated Cas9; PAM: protospacer adjacent motif; DSB: double-strand breakage; NHEJ: endogenous nonhomologous end joining; IPTG: isopropyl- $\beta$-D-thiogalactopyranoside; CRISPRi: CRISPR interference; RFP: red fluorescent protein; SGY: soya peptone-glucose-yeast extract; $\mathrm{Km}$ : kanamycin; Cm: chloramphenicol; SNPs: single nucleotide polymorphisms; CDSs: coding sequences.

\section{Authors' contributions}

$J L, Y W, J S$, and PZ conceived and designed the experiments. JL, YW, and $Y L$ performed the experiments. JL and $Y W$ analyzed the data. JS, PZ, and YM contributed reagents and analytic tools. JL, YW, JS, PZ, and YM wrote the paper. All authors read and approved the final manuscript.

\section{Author details}

${ }^{1}$ Key Laboratory of Systems Microbial Biotechnology, Chinese Academy of Sciences, Tianjin 300308, People's Republic of China. ${ }^{2}$ Tianjin Institute of Industrial Biotechnology, Chinese Academy of Sciences, Tianjin 300308, People's Republic of China.

\section{Acknowledgements}

We thank Dr. Meng Wang for helpful discussions.

\section{Competing interests}

The authors declare that they have no competing interests.

\section{Availability of data and materials}

All data generated or analyzed during this study are included in this published article and its additional files. The authors are willing to provide any additional data and materials related to this research that may be requested for research purposes.

\section{Consent for publication}

Not applicable.

\section{Ethics approval and consent to participate}

Not applicable.

\section{Funding}

This work was supported by the National Natural Science Foundation of China (31700044 and 31370829), the Key Research Program of the Chinese Academy of Sciences (ZDRW-ZS-2016-2), and the first Special Support Plan for Talents Development and High-level Innovation and Entrepreneurship Team of the Tianjin Municipal City.

\section{Publisher's Note}

Springer Nature remains neutral with regard to jurisdictional claims in published maps and institutional affiliations. 
Received: 29 June 2017 Accepted: 8 November 2017 Published online: 16 November 2017

\section{References}

1. Becker J, Gießelmann G, Hoffmann SL, Wittmann C. Corynebacterium glutamicum for sustainable bioproduction: from metabolic physiology to systems metabolic engineering. In: Zhao H, Zeng A-P, editors. Synthetic biology-metabolic engineering. Advances in Biochemical Engineering/ Biotechnology. vol 162. Cham: Springer; 2016. p. 217-63.

2. Becker J, Wittmann C. Industrial microorganisms: Corynebacterium glutamicum. In: Wittmann C, Liao JC, editors. Industrial biotechnology: microorganisms. Hoboken: Wiley; 2016. p. 183-220.

3. Cleto S, Jensen JV, Wendisch VF, Lu TK. Corynebacterium glutamicum metabolic engineering with CRISPR interference (CRISPRi). ACS Synth Biol. 2016:5:375-85

4. Vertes AA, Inui M, Yukawa H. Manipulating corynebacteria, from individual genes to chromosomes. Appl Environ Microbiol. 2005;71:7633-42.

5. Ozaki A, Katsumata R, Oka T, Furuya A. Functional expression of the genes of Escherichia coli in gram-positive Corynebacterium glutamicum. Mol Gen Genet. 1984;196:175-8.

6. Schafer A, Tauch A, Jager W, Kalinowski J, Thierbach G, Puhler A. Small mobilizable multi-purpose cloning vectors derived from the Escherichia coli plasmids pK18 and pK19: selection of defined deletions in the chromosome of Corynebacterium glutamicum. Gene. 1994;145:69-73.

7. Komor AC, Badran AH, Liu DR. CRISPR-based technologies for the manipulation of eukaryotic genomes. Cell. 2017;168:20-36.

8. Bengtsson NE, Hall JK, Odom GL, Phelps MP, Andrus CR, Hawkins RD, Hauschka SD, Chamberlain JR, Chamberlain JS. Muscle-specific CRISPR/ Cas9 dystrophin gene editing ameliorates pathophysiology in a mouse model for Duchenne muscular dystrophy. Nat Commun. 2017;8:14454.

9. Kim H, Kim ST, Ryu J, Kang BC, Kim JS, Kim SG. CRISPR/Cpf1-mediated DNA-free plant genome editing. Nat Commun. 2017;8:14406.

10. Choi KR, Lee SY. CRISPR technologies for bacterial systems: current achievements and future directions. Biotechnol Adv. 2016;34:1180-209.

11. Jiang W, Bikard D, Cox D, Zhang F, Marraffini LA. RNA-guided editing of bacterial genomes using CRISPR-Cas systems. Nat Biotechnol. 2013;31:233-9.

12. Jiang Y, Chen B, Duan C, Sun B, Yang J, Yang S. Multigene editing in the Escherichia coli genome via the CRISPR-Cas9 system. Appl Environ Microbiol. 2015:81:2506-14.

13. Pyne ME, Moo-Young M, Chung DA, Chou CP. Coupling the CRISPR/Cas9 system with lambda red recombineering enables simplified chromosomal gene replacement in Escherichia coli. Appl Environ Microbiol. 2015;81:5103-14.

14. Li Y, Lin Z, Huang C, Zhang Y, Wang Z, Tang YJ, Chen T, Zhao X. Metabolic engineering of Escherichia coli using CRISPR-Cas9 meditated genome editing. Metab Eng. 2015;31:13-21.

15. Standage-Beier $K$, Zhang Q, Wang X. Targeted large-scale deletion of bacterial genomes using CRISPR-nickases. ACS Synth Biol. 2015;4:1217-25.

16. Reisch CR, Prather KL. The no-SCAR (Scarless Cas9 Assisted Recombineering) system for genome editing in Escherichia coli. Sci Rep. 2015;5:15096.

17. Bassalo MC, Garst AD, Halweg-Edwards AL, Grau WC, Domaille DW, Mutalik VK, Arkin AP, Gill RT. Rapid and efficient one-step metabolic pathway integration in E. coli. ACS Synth Biol. 2016;5:561-8.

18. Pines G, Pines A, Garst AD, Zeitoun Rl, Lynch SA, Gill RT. Codon compression algorithms for saturation mutagenesis. ACS Synth Biol. 2015:4:604-14.

19. Chung ME, Yeh IH, Sung LY, Wu MY, Chao YP, Ng IS, Hu YC. Enhanced integration of large DNA into E. coli chromosome by CRISPR/Cas9. Biotechnol Bioeng. 2017;114:172-83.

20. Selle K, Klaenhammer TR, Barrangou R. CRISPR-based screening of genomic island excision events in bacteria. Proc Natl Acad Sci USA. 2015;112:8076-81.

21. Oh JH, van Pijkeren JP. CRISPR-Cas9-assisted recombineering in Lactobacillus reuteri. Nucleic Acids Res. 2014;42:e131

22. Cobb RE, Wang Y, Zhao H. High-efficiency multiplex genome editing of Streptomyces species using an engineered CRISPR/Cas system. ACS Synth Biol. 2015:4:723-8.
23. Huang H, Zheng G, Jiang W, Hu H, Lu Y. One-step high-efficiency CRISPR/ Cas9-mediated genome editing in Streptomyces. Acta Biochim Biophys Sin. 2015:47:231-43.

24. Tong Y, Charusanti P, Zhang L, Weber T, Lee SY. CRISPR-Cas9 based engineering of Actinomycetal genomes. ACS Synth Biol. 2015:4:1020-9.

25. Xu T, Li Y, Shi Z, Hemme CL, Li Y, Zhu Y, Van Nostrand JD, He Z, Zhou J. Efficient genome editing in Clostridium cellulolyticum via CRISPR-Cas9 nickase. Appl Environ Microbiol. 2015;81:4423-31.

26. Wang Y, Zhang ZT, Seo SO, Lynn P, Lu T, Jin YS, Blaschek HP. Bacterial genome editing with CRISPR-Cas9: deletion, integration, single nucleotide modification, and desirable "clean" mutant selection in Clostridium beijerinckii as an example. ACS Synth Biol. 2016;5:721-32.

27. Pyne ME, Bruder MR, Moo-Young M, Chung DA, Chou CP. Harnessing heterologous and endogenous CRISPR-Cas machineries for efficient markerless genome editing in Clostridium. Sci Rep. 2016;6:25666.

28. Li Q, Chen J, Minton NP, Zhang Y, Wen Z, Liu J, Yang H, Zeng Z, Ren X, Yang J, Gu Y, Jiang W, Jiang Y, Yang S. CRISPR-based genome editing and expression control systems in Clostridium acetobutylicum and Clostridium beijerinckii. Biotechnol J. 2016;11:961-72.

29. Nagaraju S, Davies NK, Walker DJ, Kopke M, Simpson SD. Genome editing of Clostridium autoethanogenum using CRISPR/Cas9. Biotechnol Biofuels. 2016:9.219

30. Westbrook AW, Moo-Young M, Chou CP. Development of a CRISPR-Cas9 tool kit for comprehensive engineering of Bacillus subtilis. Appl Environ Microbiol. 2016;82:4876-95.

31. Zhang K, Duan X, Wu J. Multigene disruption in undomesticated Bacillus subtilis ATCC 6051a using the CRISPR/Cas9 system. Sci Rep. 2016:6:27943.

32. Altenbuchner J. Editing of the Bacillus subtilis genome by the CRISPRCas9 system. Appl Environ Microbiol. 2016:82:5421-7.

33. Liu Q, Gao R, Li J, Lin L, Zhao J, Sun W, Tian C. Development of a genomeediting CRISPR/Cas9 system in thermophilic fungal Myceliophthora species and its application to hyper-cellulase production strain engineering. Biotechnol Biofuels. 2017:10:1.

34. Wendt KE, Ungerer J, Cobb RE, Zhao H, Pakrasi HB. CRISPR/Cas9 mediated targeted mutagenesis of the fast growing cyanobacterium Synechococcus elongatus UTEX 2973. Microb Cell Fact. 2016;15:115.

35. Cho JS, Choi KR, Prabowo CPS, Shin JH, Yang D, Jang J, Lee SY. CRISPR/ Cas9-coupled recombineering for metabolic engineering of Corynebacterium glutamicum. Metab Eng. 2017;42:157-67.

36. Jiang Y, Qian F, Yang J, Liu Y, Dong F, Xu C, Sun B, Chen B, Xu X, Li Y, Wang R, Yang S. CRISPR-Cpf1 assisted genome editing of Corynebacterium glutamicum. Nat Commun. 2017:8:15179.

37. Zetsche B, Gootenberg JS, Abudayyeh OO, Slaymaker IM, Makarova KS, Essletzbichler P, Volz SE, Joung J, van der Oost J, Regev A, Koonin EV, Zhang F. Cpf1 is a single RNA-guided endonuclease of a class 2 CRISPRCas system. Cell. 2015;163:759-71.

38. Peyret JL, Bayan N, Joliff G, Gulik-Krzywicki T, Mathieu L, Schechter E, Leblon G. Characterization of the cspB gene encoding PS2, an ordered surface-layer protein in Corynebacterium glutamicum. Mol Microbiol. 1993:9:97-109.

39. Qi LS, Larson MH, Gilbert LA, Doudna JA, Weissman JS, Arkin AP, Lim WA Repurposing CRISPR as an RNA-guided platform for sequence-specific control of gene expression. Cell. 2013:152:1173-83.

40. Resende BC, Rebelato AB, D'Afonseca V, Santos AR, Stutzman T, Azevedo VA, Santos LL, Miyoshi A, Lopes DO. DNA repair in Corynebacterium model. Gene. 2011;482:1-7.

41. Ellis HM, Yu D, DiTizio T, Court DL. High efficiency mutagenesis, repair, and engineering of chromosomal DNA using single-stranded oligonucleotides. Proc Natl Acad Sci USA. 2001;98:6742-6.

42. Binder S, Siedler S, Marienhagen J, Bott M, Eggeling L. Recombineering in Corynebacterium glutamicum combined with optical nanosensors: a general strategy for fast producer strain generation. Nucleic Acids Res. 2013;41:6360-9.

43. Hsu PD, Scott DA, Weinstein JA, Ran FA, Konermann S, Agarwala V, LiY, Fine EJ, Wu X, Shalem O, Cradick TJ, Marraffini LA, Bao G, Zhang F. DNA targeting specificity of RNA-guided Cas9 nucleases. Nat Biotechnol. 2013;31:827-32.

44. Krylov AA, Kolontaevsky EE, Mashko SV. Oligonucleotide recombination in corynebacteria without the expression of exogenous recombinases. $J$ Microbiol Methods. 2014;105:109-15. 
45. Kim IK, Jeong WK, Lim SH, Hwang IK, Kim YH. The small ribosomal protein S12P gene rps $L$ as an efficient positive selection marker in allelic exchange mutation systems for Corynebacterium glutamicum. J Microbiol Methods. 2011;84:128-30

46. Nakamura J, Kanno S, Kimura E, Matsui K, Nakamatsu T, Wachi M. Temperature-sensitive cloning vector for Corynebacterium glutamicum. Plasmid. 2006:56:179-86.

47. Nesvera J, Patek M, Hochmannova J, Abrhamova Z, Becvarova V, Jelinkova M, Vohradsky J. Plasmid pGA1 from Corynebacterium glutamicum codes for a gene product that positively influences plasmid copy number. J Bacteriol. 1997;179:1525-32

48. Nakamura Y, Nishio Y, Ikeo K, Gojobori T. The genome stability in Corynebacterium species due to lack of the recombinational repair system. Gene. 2003;317:149-55.

49. Leenay RT, Maksimchuk KR, Slotkowski RA, Agrawal RN, Gomaa AA, Briner $A E$, Barrangou R, Beisel CL. Identifying and visualizing functional PAM diversity across CRISPR-Cas systems. Mol Cell. 2016;62:137-47.

50. Bartoszewski RA, Jablonsky M, Bartoszewska S, Stevenson L, Dai Q, Kappes J, Collawn JF, Bebok Z. A synonymous single nucleotide polymorphism in $\triangle F 508$ CFTR alters the secondary structure of the mRNA and the expression of the mutant protein. J Biol Chem. 2010;285:28741-8.
51. Cui L, Bikard D. Consequences of Cas9 cleavage in the chromosome of Escherichia coli. Nucleic Acids Res. 2016:44:4243-51.

52. Ma W, Wang X, Mao Y, Wang Z, Chen T, Zhao X. Development of a markerless gene replacement system in Corynebacterium glutamicum using upp as a counter-selection marker. Biotechnol Lett. 2015;37:609-17.

53. Ruan Y, Zhu L, Li Q. Improving the electro-transformation efficiency of Corynebacterium glutamicum by weakening its cell wall and increasing the cytoplasmic membrane fluidity. Biotechnol Lett. 2015;37:2445-52.

54. Zhao D, Yuan S, Xiong B, Sun H, Ye L, Li J, Zhang X, Bi C. Development of a fast and easy method for Escherichia coli genome editing with CRISPR/ Cas9. Microb Cell Fact. 2016;15:205.

55. Kirchner O, Tauch A. Tools for genetic engineering in the amino acidproducing bacterium Corynebacterium glutamicum. J Biotechnol. 2003;104:287-99.

56. Jakoby M, Ngouoto-Nkili C-E, Burkovski A. Construction and application of new Corynebacterium glutamicum vectors. Biotechnol Tech. 1999;13:437-41.

\section{Submit your next manuscript to BioMed Central and we will help you at every step:}

- We accept pre-submission inquiries

- Our selector tool helps you to find the most relevant journal

- We provide round the clock customer support

- Convenient online submission

- Thorough peer review

- Inclusion in PubMed and all major indexing services

- Maximum visibility for your research

Submit your manuscript at www.biomedcentral com/submit 